REPRESENTATION THEORY

An Electronic Journal of the American Mathematical Society

Volume 15, Pages 385-406 (May 3, 2011)

S 1088-4165(2011)00401-5

\title{
QUOTIENTS OF REPRESENTATION RINGS
}

\author{
HANS WENZL
}

\begin{abstract}
We give a proof using so-called fusion rings and $q$-deformations of Brauer algebras that the representation ring of an orthogonal or symplectic group can be obtained as a quotient of a ring $\operatorname{Gr}(O(\infty))$. This is obtained here as a limiting case for analogous quotient maps for fusion categories, with the level going to $\infty$. This in turn allows a detailed description of the quotient map in terms of a reflection group. As an application, one obtains a general description of the branching rules for the restriction of representations of $G l(N)$ to $O(N)$ and $S p(N)$ as well as detailed information about the structure of the $q$-Brauer algebras in the nonsemisimple case for certain specializations.
\end{abstract}

It is well known that one can study the combinatorics of the finite dimensional representations of the general linear groups $G l(N)$ in a uniform way essentially independently of the dimension $N$, using the representation theory of the symmetric groups. A similar approach is possible for orthogonal and symplectic groups. In particular, it is possible to obtain their Grothendieck rings as quotients from a large ring, denoted here by $\operatorname{Gr}(O(\infty)$ ) (see e.g. [KT]). More recently, quotients $\operatorname{Gr}(G)_{\ell}$ of the Grothendieck ring $G r(G)$ of a semisimple Lie group $G$, depending on a positive integer $\ell$, have been studied which have only finitely many simple objects up to isomorphism. They originally arose in mathematical physics and are usually called fusion rings. One of the observations in this paper is that there is more than just a formal similarity between these two quotients:

We show that the quotient map from $\operatorname{Gr}(O(\infty))$ onto the Grothendieck ring $\operatorname{Gr}(S p(N))$ of a symplectic group can be obtained as a limit of the quotient map $G r(S p(M))$ to $\operatorname{Gr}(S p(M))_{\ell(M)}$ for $M \rightarrow \infty$; here $\ell(M)$ depends on $M$ in a simple linear way. A similar result also holds for orthogonal groups. This allows, among other things, for a simple explicit description of the quotient map in terms of a reflection group. One of the applications is an explicit formula for the restriction multiplicities for representations from $G l(N)$ to $O(N)$ and to $S p(N)$, respectively; see below for more details and related results. As another application, we also obtain results about the structure of a $q$-deformation $\hat{\mathcal{C}}_{n}(r, q)$ of Brauer's centralizer algebras $\mathcal{C}_{n}(x)$ when $r=q^{-N-1}$, with $N>0$ even. In our approach, we get these results as a limiting case of rather deep results about tilting modules of quantum groups. This also makes the appearance of parabolic Kazhdan-Lusztig polynomials fairly natural.

Here is the contents of our paper in more detail. The first section reviews results about so-called fusion rings, which are certain quotients of the representation

Received by the editors December 11, 2006 and, in revised form, January 11, 2011.

2010 Mathematics Subject Classification. Primary 22E46.

This work was partially supported by NSF grant DMS 0302437.

(C)2011 American Mathematical Society Reverts to public domain 28 years from publication 
rings of semisimple Lie algebras, depending on a positive integer $\ell$. This is fairly elementary. We can deduce from this one of our main results, the already mentioned explicit description of the quotient maps from $\operatorname{Gr}(O(\infty))$ onto $\operatorname{Gr}(\operatorname{Sp}(N))$ and $\operatorname{Gr}(O(N))$, respectively. The only result which is not proved by elementary methods is the so-called level-rank duality, see Proposition 1.8, A very simple proof can be given after expressing the corresponding fusion rings in terms of $q$-versions of Brauer's centralizer algebras. Section 2 reviews the necessary results about these algebras. In Section 3 we define semirings in terms of these algebras and eventually show these semirings are related to the fusion rings defined in the first section. Section 4 then proves the restriction rules from $G l(N)$ to $O(N)$ and $S p(N)$, respectively. Finally, in Section 5, we first review how results by Soergel and $\mathrm{Hu}$ determine the structure of the image of the algebras $\hat{\mathcal{C}}_{n}\left(q^{-N-1}, q\right), N$ even, in a certain tensor space. Using the already mentioned level-rank duality, we extend this to all of the algebras in the generic case, i.e., for all but finitely many values of $q$.

This paper deals with some very familiar and heavily studied subjects. So, not surprisingly, many related results have appeared before. We just mention a few: This paper was motivated by the paper [EW] where restriction rules have been studied using a completely different approach. After the first version of our paper was finished, we also learned of the work [KT] with very similar results, and an approach much closer to the one used here, and other combinatorial approaches, including [Ki] and $[\mathrm{Su}$. Nevertheless, we think that the connection to fusion rings and tilting modules in our paper provides additional insight. In the version of the paper which was first submitted in December 2006, there was also a conjecture about the structure of Brauer's centralizer algebra in the nonsemisimple case, inspired by Soergel's work on tilting modules. This conjecture was also obtained, independently, in CdVM] and, more recently, a proof has been posted in [Mt]. In addition, the paper $\mathrm{Hu}$. has been published, which proves one of the assumptions for our approach. We decided to replace the section dealing with the conjecture with a new section which does prove the conjecture in a special case for the $q$ deformation of the Brauer algebras. This should give the same result as the one in $\mathrm{Mt}$ if $q \rightarrow 1$, but the method would be completely different. The rest of the paper has the same results as the first version. Some substantial changes have been made in the hope of making the presentation more conceptual and clearer, but no new material has been used for that.

\section{FUSION RINGS}

1.1. General construction. The material here is well known. For some background, see e.g. the books [FMS] and [Kc]. Let $G$ be a compact simple Lie group. Let $\mathfrak{g}$ be its Lie algebra with Cartan subalgebra $\mathfrak{h}$, and let $\mathfrak{h}^{*}$ be the linear dual of $\mathfrak{h}$. As in $\overline{\mathrm{Kc}}$ we denote the coroot lattice by $M$. We normalize the invariant bilinear form on $\mathfrak{h}^{*}$ such that $(\alpha, \alpha)=2$ for a long root $\alpha$. Identifying $\mathfrak{h}^{*}$ with $\mathfrak{h}$ via this form, we can assume that the coroot lattice $M$ is a sublattice of the weight lattice $M^{*}$. Moreover, the weight lattice is given by $M^{*}=\left\{\mathbf{y} \in \mathfrak{h}^{*},(\mathbf{y}, \mathbf{x}) \in \mathbb{Z}\right.$ for all $\mathbf{x} \in M\}$. For a given dominant weight $\lambda$, we denote by $V_{\lambda}$ a simple $\mathfrak{g}$ module with highest weight $\lambda$, and by $\left[V_{\lambda}\right]$ its equivalence class in the Grothendieck semiring of $\mathfrak{g}$. We will explicitly write $M$ and $M^{*}$ for the Lie algebra of an orthogonal or a symplectic group below. 
For any $\mathbf{y} \in \mathfrak{h}^{*}$ we obtain a multiplicative character $e^{\mathbf{y}}$ of $\mathfrak{h}$ via $e^{\mathbf{y}}(\mathbf{x})=$ $\exp (2 \pi i(\mathbf{y}, \mathbf{x}))$. Fix $\ell \in \mathbb{N}$ and let $\ell^{-1} M^{*}=\left\{\mathbf{y} \in \mathfrak{h}, \ell \mathbf{y} \in M^{*}\right\}$. Then $\ell^{-1} M^{*} / M$ is a finite group whose simple characters are given by $\left\{e^{\mathbf{y}}, \mathbf{y} \in M^{*} / \ell M\right\}$. Let $W$ be the Weyl group associated to $G$. We define, as usual, the Weyl character $\chi^{\lambda}(\mathbf{x})=\left(\sum_{w \in W} \varepsilon(w) e^{w(\lambda+\rho)}(\mathbf{x})\right) /\left(\sum_{w \in W} \varepsilon(w) e^{w(\rho)}(\mathbf{x})\right)$, where $\rho$ is half the sum of the positive roots of $G$. Let $W^{(l)}$ be the associated affine reflection group. The latter is the semidirect product of $W$ with $\ell M$, where the latter group acts via translations $t_{\mathbf{x}} \mathbf{y}=\mathbf{y}+\mathbf{x}$ on $\mathfrak{h}^{*}$. It is well known that $W^{(l)}$ has a fundamental domain $\bar{D}_{\ell}$, the closure of the set $D_{\ell}$ consisting of elements $\mathbf{y} \in \mathfrak{h}^{*}$ satisfying $\left(\alpha_{i}, \mathbf{y}\right)>0$ and $(\theta, \mathbf{y})<\ell$; here the $\alpha_{i}$ are the simple roots of $G$, and $\theta$ is the maximum long root of $G$. It is well known that $W^{(l)}$ is a reflection group, generated by the reflection $s_{0}$ in the hyperplane given by $(\theta, \mathbf{y})=\ell$ and the simple reflections of $W$. The hyperplanes for these generating reflections contain the boundary simplices of $D_{\ell}$. We define a second action of $W^{(l)}$ on $\mathfrak{h}^{*}$ by $w \cdot \lambda=w(\lambda+\rho)-\rho$ for $\lambda \in \mathfrak{h}^{*}$.

Proposition 1.1. Let $G$ be a connected and simply connected simple Lie group. Let $I_{\ell}(G)$ be the ideal in the representation ring of $G$ generated by all those characters which annihilate $\ell^{-1} M^{*}$. Then $\operatorname{Gr}(G)_{\ell}=G r(G) / I_{\ell}(G)$ has a $\mathbb{Z}$-basis $\left(\left[V_{\lambda}\right]\right)$ labeled by all those dominant weights $\lambda$ for which $\lambda+\rho$ is in $D_{\ell}$. Moreover, we have $\left[V_{w \cdot \lambda}\right]=\varepsilon(w)\left[V_{\lambda}\right]$ modulo $I_{\ell}(G)$ for any $w \in W^{(l)}$ which maps the dominant weight $\lambda$ to a dominant weight w. $\lambda$. The spectrum of the abelian ring $G r(G)_{\ell}$ is given by the maps $\phi_{\mu}:\left[V_{\lambda}\right] \mapsto \chi^{\lambda}((\mu+\rho) / \ell)$, where $\mu$ goes over all dominant weights of $G$ such that $\mu+\rho \in D_{\ell}$.

Proof. This is well known. We give simple arguments for the reader's convenience. Define for $\mathbf{y} \in M^{*}$ the map $a_{W}\left(e^{\mathbf{y}}\right)=\sum_{w \in W} \varepsilon(w) e^{w(\mathbf{y})}$. If $\lambda$ is a dominant weight, $a_{W}\left(e^{\lambda+\rho}\right)$ is the numerator in the Weyl character formula for the simple module with highest weight $\lambda$. Moreover, for such $\lambda$, we can find $\mathbf{x} \in \ell M$ and $w \in W$ such that $w t_{\mathbf{x}}(\lambda+\rho)$ is in $\overline{D_{\ell}}$ (the reader not familiar with this should have little problem checking this for the relevant cases which will be listed below). It follows that $a_{W}\left(e^{\lambda+\rho}\right)=\varepsilon\left(w t_{\mathbf{x}}\right) a_{W}\left(e^{w t_{\mathbf{x}}(\lambda+\rho)}\right)$; the latter expression is zero if $w t_{\mathbf{x}}(\lambda+\rho)$ is not in $D_{\ell}$, i.e., if it lies on one of its boundary simplices.

Finally, it is clear that each $\phi_{\mu}$ defines a homomorphism from $G r(G)_{\ell}$ into $\mathbb{C}$. Let $d(\mu)=\chi^{\mu}(\rho / \ell)$ be the so-called $q$-dimension of $V_{\mu}$. Then $s_{\lambda \mu}=\chi^{\lambda}((\mu+\rho) / \ell) d(\mu)$ is an entry of the $S$-matrix $S=\left(s_{\lambda \mu}\right)$ in $[\mathrm{KP}$. It is shown there that this matrix is invertible (or see, e.g. [TW2], Prop. 9.8.1 for the simple argument). Hence the $\phi_{\mu}$ are linearly independent and they exhaust all one-dimensional characters of $\operatorname{Gr}(G)_{\ell}$.

Remark 1.2. 1. We define $G r(G)_{\ell,+}$ to be the $\mathbb{N}$-linear span of the elements $\lambda \in$ $\operatorname{Gr}(G)_{\ell}$ for which $\lambda+\rho$ is in $D_{\ell}$. We shall see later that this does indeed define a semiring.

2. With some care, one can also similarly define quotients $G r(G)_{\ell}$ if $G$ is not necessarily connected or simply connected. This will be done below for orthogonal groups.

1.2. Orthogonal case. Let us first explicitly describe the set-up of the last subsection for the odd-dimensional orthogonal Lie algebra, i.e., for Lie type $B_{m}$. For type $B_{m}$, we can take $M=\left\{\mathbf{x} \in \mathbb{Z}^{m}, 2 \mid \sum x_{i}\right\}$ and $M^{*}=\mathbb{Z}^{m} \cup\left(\mathbf{j}+\mathbb{Z}^{m}\right)$, where $\mathbf{j}=(1 / 2,1 / 2, \ldots, 1 / 2) \in \mathbb{R}^{m}$. Here the invariant inner products on $M$ and $M^{*}$ 
are given by the usual scalar product on $\mathbb{R}^{m}$. Its Weyl group $W(m)$ is isomorphic to the semidirect product of $(\mathbb{Z} / 2)^{m}$ with the symmetric group $S_{m}$. It has a natural action on $\mathfrak{h}^{*} \cong \mathbb{R}^{m}$ where $S_{m}$ permutes the coordinates and $(\mathbb{Z} / 2)^{m}$ acts via possible sign changes on the coordinates. Its maximum longest root is given by $\theta(\mathbf{x})=x_{1}+x_{2}$ for $\mathbf{x} \in \mathbb{R}^{m}$. The corresponding affine Weyl group $W^{(l)}(m)$ has as fundamental domain the closure of the set $D_{\ell}=\left\{x \in \mathbb{R}^{m}, x_{1}>x_{2}>\ldots x_{m}>0\right.$ with $\left.x_{1}+x_{2}<\ell\right\}$. Finally, we set $\rho(m)$ to be equal to half of the sum of all positive roots; in our notation, that means $\rho(m)=\left(m+\frac{1}{2}-i\right)_{i} \in \mathbb{R}^{m}$.

For the Lie algebra $s o_{2 m}$, we have the same lattices $M$ and $M^{*}$ and the same longest root $\theta$ as for Lie type $B_{m}$. The Weyl group $W(m)$ now is isomorphic to the semidirect product $(\mathbb{Z} / 2)^{m-1} \ltimes S_{m}$, where $(\mathbb{Z} / 2)^{m-1}$ is identified with the subgroup of $(\mathbb{Z} / 2)^{m}$ consisting of elements with an even number of nonzero entries. We define the set $D_{\ell}$ for the affine Weyl group $W^{(\ell)}(m)$ as for type $B_{m}$ except for the inequality $\ldots>x_{m-1}>\left|x_{m}\right|$; its closure is again a fundamental domain.

1.3. The semiring $\operatorname{Gr}(O(\infty))_{+}$. Let $M$ and $M^{*}$ be as in the last subsection, for the odd-dimensional case $S O(2 m+1)$. Only the weights in $M^{*}$ with integer coefficients are also weights of the corresponding orthogonal group. These weights are invariant under the action of the group $W^{(l)}(m)$, hence we obtain a well-defined subgroup of the quotient $\operatorname{Gr}(\operatorname{Spin}(2 m+1))_{\ell}$, as defined in Proposition 1.1 In particular, the dominant weights are given by vectors with nonnegative nonincreasing integer coefficients. We shall often identify such a dominant weight $\lambda$ with a Young diagram, denoted by the same symbol, which has $\lambda_{i}$ boxes in its $i$-th row. This allows an obvious injection of the dominant weights of $S O(2 m+1)$ into the dominant weights of $S O(2(m+1)+1)$. It turns out that for given dominant weights $\lambda$ and $\mu$ these embeddings are compatible with the tensor product rules for large enough $m$. More precisely, we have the following well-known proposition (see e.g. [KT]; we will give another proof in Lemma 3.3).

Proposition 1.3. Let $V_{\lambda}, V_{\mu}$ be simple $S O(2 m+1)$ modules labeled by Young diagrams $\lambda$ and $\mu$, and let $V_{\lambda} \otimes V_{\mu}=\bigoplus d_{\lambda \mu}^{\nu}(m) V_{\nu}$ be the decompostion of the tensor product into a direct sum of simple modules. Then $d_{\lambda \mu}^{\nu}(m)=d_{\lambda \mu}^{\nu}$ independently of $m$ for $m$ sufficiently large. In particular, we can define a semiring $\operatorname{Gr}(O(\infty))_{+}$ with an $\mathbb{N}$ basis $\{[\lambda]\}$ labeled by Young diagrams whose multiplication is given by the structure coefficients $d_{\lambda \mu}^{\nu}$.

1.4. Limiting reflection group. With the notation of the last section for type $B_{m}$, let $\ell=N+2 m-1$ for $N$ be a fixed positive integer. It will turn out that we can construct a limiting Grothendieck ring for $m \rightarrow \infty$ similarly as we defined the ring $\operatorname{Gr}(O(\infty))$ in the last section. It will turn out that for given $\lambda$ and large $m$, only certain reflections of $W^{(l)}(m)$ will be relevant to map $\lambda$ into $D_{\ell}$. Using only these reflections, with the standard embedding $W(m) \subset W(m+1)$, we obtain a limiting reflection group $W=W(\infty)$. This group $W$ has Coxeter type $D_{\infty}$. It is generated by the group $S_{\infty}$ of finite permutations on a countable set together with one additional reflection $s_{o}$. It can be conveniently described by a faithful representation on the space of sequences, on which $S_{\infty}$ acts via permutations of the entries of the sequence and where the reflection $s_{0}$ acts by $s_{0}\left(l_{1}, l_{2}, l_{3}, \ldots\right)=$ $\left(-l_{2},-l_{1}, l_{3}, \ldots\right)$. In particular, we can define the sign $\varepsilon(w)$ of an element $w \in W$ to be $(-1)^{k}$, where $k$ is the number of factors if we write $w$ as a product of simple 
reflections. Let $\rho$ be the sequence $(1-N / 2-i)_{i \in \mathbb{N}}$. Then we define a second action on sequences $\lambda$ as before by $w \cdot \lambda=w(\lambda+\rho)-\rho$.

We also define $D(N)$ to be the set of all Young diagrams with $\leq N$ boxes in the first two rows. Similarly, as $\operatorname{Gr}(G)$ can be obtained as a quotient of $\operatorname{Gr}(O(\infty))$, we can now define a ring which contains all the orthogonal fusion rings as quotients. The algorithm in the proof below has already appeared; see $\underline{\mathrm{Su}}$.

Lemma 1.4. Let $N$ be a fixed positive integer, and let $\ell=N+2 m-1$, with $m$ also being a positive integer. Let $\lambda \in \Lambda$ be a Young diagram with $\leq m$ rows.

(a) There exists an element $w \in W$ such that either $w \cdot \lambda \in D(N)$ or $\lambda+\rho$ is fixed by some reflection in $W$. Moreover, if $w . \lambda \in D(N)$, it is contained in $\lambda$, i.e. $(w . \lambda)_{i} \leq \lambda_{i}$ for all $i$.

(b) If $\lambda_{1}+\lambda_{2}<2(N+m+1)$ and if $\tilde{w} \in W^{(l)}(m)$ is such that $\tilde{w} \cdot \lambda \in D_{N}$, then $\tilde{w} \cdot \lambda=w \cdot \lambda$, with $w$ as in (a); in particular, $\tilde{w} \cdot \lambda$ does not depend on $m$.

Proof. Let us first consider the situation in part (b). Set $\mathbf{x}=\lambda+\rho(m)$. There is nothing to show if $x_{1}+x_{2} \leq \ell$. By assumption, we have $x_{1}+x_{2}<2 \ell$. Hence, if $\mathbf{y}=$ $s_{0}(\mathbf{x})$, we have $0<\mathbf{y}_{1}+\mathbf{y}_{2}<\mathbf{x}_{1}+\mathbf{x}_{2}$. If $\mathbf{y}$ does not have two identical coordinates (which does imply that $\mathbf{x}$ is fixed by a reflection), we can apply a permutation $w$ to y such that $w(\mathbf{y})$ has strictly decreasing coordinates, i.e., $w(\mathbf{y})-\rho=\tilde{\lambda}$ is a Young diagram with $\tilde{\lambda}_{i} \leq \lambda_{i}$ for all $i$ and $\tilde{\lambda}_{1}+\tilde{\lambda}_{2}<\lambda_{1}+\lambda_{2}$. Iterating this process, we will end up with a diagram as described in part (a).

To prove the remaining parts of this lemma, it suffices to check that $s_{0} \cdot \lambda=$ $\lambda-\left(\lambda_{1}+\lambda_{2}-N-1\right)(1,1,0, \ldots)$ both for sufficiently large $m$ as well as for $W=W(\infty)$. This is straightforward. Hence the algorithm in the last paragraph works as well in the setting of $W=W(\infty)$, and yields the same result.

Proposition 1.5. For each $N \in \mathbb{N}$ there exists an ideal $\mathcal{I}_{N}=\mathcal{I}_{O(N)}$ such that the quotient $\operatorname{Gr}(O(\infty)) / \mathcal{I}_{N}$ of $G r(O(\infty))$ has a $\mathbb{Z}$-basis labelled by the set $D(N)$ of Young diagrams $\lambda$ satisfying $\lambda_{1}+\lambda_{2} \leq N$. For other Young diagrams $\mu$, we either have $[\mu] \in \mathcal{I}_{N}$, or there exists a $w \in W$ such that $w . \mu \in D(N)$ and $[\mu] \cong \varepsilon(w)[w . \mu]$ $\bmod \mathcal{I}_{N}$.

Proof. We have seen that the product $[\lambda][\mu]$ of elements $[\lambda],[\mu] \in G r(O(\infty))_{+}$is given by the decomposition of the tensor product $V_{\lambda} \otimes V_{\mu}$ of irreducible $S O(2 m+1)$ modules, for $m$ sufficiently large. It only remains to show that the reduction modulo $\mathcal{I}_{N}(m)$ does not depend on $m$ provided $m$ is sufficiently large. But it follows from the algorithm in the proof of Lemma 1.4 that the fact whether $[\lambda] \in \mathcal{I}_{N}(m)$ and, if not, the value of $w \cdot \lambda \in D(N)$ is independent of $m$, provided it is large enough.

1.5. Symplectic case. In the symplectic case, Lie type $C_{m}$, we can take $M=2 \mathbb{Z}^{m}$ and $M^{*}=\mathbb{Z}^{m}$, with the inner product given by one half of the usual scalar product on $\mathbb{R}^{m}$. The Weyl group $W(m)$ is the same as for type $B_{m}$, while the maximum long root $\theta$ now is given by $\theta(\mathbf{x})=x_{1}$. We again define the affine reflection group $W^{(l)}(m)$ as the semidirect product of $\ell M$ with $W$. It now has a fundamental domain given by the closure of the set $D_{\ell}=\left\{x \in \mathbb{R}^{m}, x_{1}>x_{2}>\ldots x_{m}>0\right.$ with $\left.x_{1}<\ell\right\}$. In this setting, half the sum of the positive roots is equal to $\rho(m)=(m+1-i)_{i} \in \mathbb{R}^{m}$.

We will again define a limiting reflection group $W=W(\infty)$, this time of type $B_{\infty}$, which is generated by $S_{\infty}$ and an additional reflection $s_{0}$. It has a faithful action on the set of sequences given by $s_{o}\left(l_{1}, l_{2}, \ldots\right)=\left(-l_{1}, l_{2}, \ldots\right)$ and by the obvious action of $S_{\infty}$. We also define $\rho$ to be the sequence $\rho=(N / 2-i)_{i}$. 
Proposition 1.6. For each even $N \in \mathbb{N}$ there exists an ideal $\mathcal{I}_{N}=\mathcal{I}_{S p(N)}$ such that the quotient $\operatorname{Gr}(O(\infty)) / \mathcal{I}_{N}$ of $G r(O(\infty))$ has a $\mathbb{Z}$-basis labelled by the set $D(N)=D(S p(N))$ of Young diagrams $\lambda$ satisfying $\lambda_{1} \leq N / 2$. For other Young diagrams $\mu$, we either have $[\mu] \equiv 0 \bmod \mathcal{I}_{N}$, or $w \in W$ exists such that $w . \mu \in D(N)$ and $[\mu] \cong \varepsilon(w)[w \cdot \mu] \bmod \mathcal{I}_{N}$.

Proof. The proof follows exactly the same pattern as the one for Proposition 1.5 . So we only give an outline of the proof. One defines ideals $\mathcal{I}_{N}(m)$ for the symplectic groups $S p(2 m)$ with $\ell=N / 2+m+1$ as in Proposition 1.1. One then proves the analog of Lemma 1.4 in this setting.

1.6. Full orthogonal groups. We will need to extend the discussion above for connected groups to the full orthogonal group $O(M)$, which is a semidirect product of $S O(M)$ with $\mathbb{Z} / 2$. Its simple modules are labeled by Young diagrams with at most $M$ boxes in the first two columns; using the notation $\lambda_{i}^{\prime}$ for the number of boxes in the $i$-th column, we can formulate this condition by $\lambda_{1}^{\prime}+\lambda_{2}^{\prime} \leq M$. We define the map $t$ on Young diagrams by $t(\lambda)_{1}^{\prime}=M-\lambda_{1}^{\prime}$ and $t(\lambda)_{i}^{\prime}=\lambda_{i}^{\prime}$ for $i>1$. Observe that $t$ defines a permutation of the irreducible representations of $O(M)$ of order two. We can now easily describe the restriction rule from $O(M)$ to $S O(M)$ as follows:

(i) If $t(\lambda) \neq \lambda$, the irreducible $O(M)$ modules $V_{\lambda}$ and $V_{t(\lambda)}$ labeled by $\lambda$ and $t(\lambda)$, respectively, are isomorpic irreducible $S O(M)$-modules. One of $\lambda$ or $t(\lambda)$ will have less than $M / 2$ rows and will give us the highest weight of the $S O(M)$-module $V_{\lambda}$.

(ii) If $t(\lambda)=\lambda$, then $M$ is even and $\lambda$ has exactly $M / 2$ rows. In this case the irreducible $O(M)$ module $V_{\lambda}$ decomposes into the direct sum of two irreducible $S O(M)$ modules with highest weights $\lambda$ and $\tilde{\lambda}$, where $\tilde{\lambda}$ coincides with $\lambda$ except for the $m$-th coordinate, which is equal to $-\lambda_{m}$.

We can now extend the quotient map from $\operatorname{Gr}(S O(M))$ to $\operatorname{Gr}(O(M))$ as follows:

Lemma 1.7. The ring $\operatorname{Gr}(O(M))$ has a quotient $G r(O(M))_{\ell}$ with a $\mathbb{Z}$ basis given by Young diagrams $\lambda$ with $\leq M$ boxes in the first two columns, and with $\leq \ell+$ $2-M$ boxes in the first two rows. This extends the quotients constructed above for $\operatorname{Gr}(S O(M))$, with the same restriction rules. In particular, the quotient map is determined by the same group $W$ as in the $S O(M)$ case, which now acts only on the first $m$ rows.

Proof. If $M$ is odd, $O(M) \cong S O(M) \times \mathbb{Z} / 2$. The action of the generator of $\mathbb{Z} / 2$ on $V_{\lambda}$ is given by the scalar $(-1)^{|\lambda|}$. It is easy to see that this is compatible with the action of the group $W$. The case is more complicated for $M=2 m$ even. If $\lambda$ has less than $m$ rows, we can apply $W$ as in the odd case. If $\lambda$ has exactly $m$ rows, it can happen that $w \cdot \lambda$ may have less than $m$ rows. In this case, it follows from the character formula that $[\lambda] \cong \varepsilon(w)([w \cdot \lambda]+[t(w \cdot \lambda)])$. Finally, if $\lambda$ has more than $m$ rows, we have $[w \cdot \lambda]=[t(w \cdot(t(\lambda)))]$.

Proposition 1.8 (Level-rank duality). The transpose map $\lambda \mapsto \lambda^{\prime}$, where $\lambda^{\prime}$ is the transposed Young diagram obtained from $\lambda$, induces an isomorphism between $G r(O(N))_{\ell}$ and $G r(O(\ell+2-N))_{\ell}$ and between $G r(S p(N))_{\ell}$ and $G r(S p(2 \ell-2-N))_{\ell}$.

This proposition will be shown at the end of Section 3 , see a brief discussion about this result at the beginning of that section. For the following theorem, we define the transposed action of $w \in W$ on a Young diagram by $w^{\prime} \cdot \lambda=\left(w \cdot \lambda^{\prime}\right)^{\prime}$, i.e., we define the action via the columns instead of the rows. 
Theorem 1.9. The transpose map $\lambda \mapsto \lambda^{\prime}$ induces an isomorphism between the rings $\operatorname{Gr}(O(N))$ and $\operatorname{Gr}(O(\infty)) / \mathcal{I}_{O(N)}$. In particular, $\operatorname{Gr}(O(N))$ is a quotient of $\operatorname{Gr}(O(\infty))$, with the quotient map explicitly given by Proposition 1.5 with respect to the transposed action of $W$. Similarly, we have isomorphisms between $\operatorname{Gr}(\operatorname{Sp}(N))$ and a quotient $\operatorname{Gr}(O(\infty)) / \mathcal{I}_{S p(N)}$, using the notation of Section 1.5 ,

Proof. The transpose maps $\operatorname{Gr}(O(\infty)) / \mathcal{I}_{O(N)}$ to a quotient of $G r(O(\infty))$ which has a basis labeled by the same Young diagrams, which also labels the irreducible representations of $O(N)$. By definition of $G r(O(\infty))$ and $G r(O(\infty)) / \mathcal{I}_{O(N)}$, the multiplication of two elements $[\lambda]$ and $[\mu]$ in the latter quotient is already determined by the one in $\operatorname{Gr}(O(\ell+2-N))_{\ell}$ for $\ell$ sufficiently large; see Lemma 1.4 and Proposition 1.5. By Proposition 1.8, the latter semiring is isomorphic to $\operatorname{Gr}(O(N))_{\ell}$. Again, for $\ell$ sufficiently large, the product is given by the usual tensor product rules of $O(N)$. The same proof works as well in the symplectic case.

\section{BRAUER ALGEBraS}

2.1. Basics (See $[\mathrm{Br}]$ ). Let $V=\mathbb{C}^{N}$ be the vector representation of the orthogonal group $O(N)$. Then, using the usual bilinear form on $V=\mathbb{C}^{N}$, we can identify $V^{*}$ with $V$, and $\operatorname{End}\left(V^{\otimes n}\right)$ with $\left(V^{*}\right)^{\otimes 2 n} \cong\left(V^{\otimes n}\right)^{*}$. Moreover, under this isomorphism, the linear space $\operatorname{End}_{O(N)}\left(V^{\otimes n}\right)$ of linear maps commuting with the $O(N)$ action on $V^{\otimes n}$ is isomorphic to the linear space of $O(N)$-invariant elements in $\left(V^{\otimes n}\right)^{*}$. It was shown by Brauer that this space is spanned by all possible maps obtained by forming the product of $n$ inner products of the $2 n$ tensor factors of an element in $V^{\otimes 2 n}$. As an example, for $n=2$, we have three such maps, which map a vector $v_{1} \otimes v_{2} \otimes v_{3} \otimes v_{4}$ to the products $\left(v_{1}, v_{3}\right)\left(v_{2}, v_{4}\right),\left(v_{1}, v_{4}\right)\left(v_{2}, v_{3}\right)$ and $\left(v_{1}, v_{2}\right)\left(v_{3}, v_{4}\right)$, respectively. In general, these maps are given by a partition of the set $\{1,2, \ldots, 2 n\}$ into $n$ disjoint subsets $\left\{i_{r}, j_{r}\right\}, 1 \leq r \leq n$ which describe the map

$$
v_{1} \otimes v_{2} \otimes \ldots \otimes v_{2 n} \quad \mapsto \prod_{r=1}^{n}\left(v_{i_{r}}, v_{j_{r}}\right) .
$$

2.2. Definitions (See $[\mathrm{Br}$, and also $[\mathrm{W} 3$ ). The discussion in the previous section motivated the definition of an abstract algebra $\mathcal{C}_{n}$ which can be defined over the ring $\mathbb{Z}[x]$ of polynomials with integer coefficients; we will usually define it over the rational functions $\mathbb{Q}(x)$. For each map in (2.1) we define a graph with $2 n$ vertices and $n$ edges, where we put the first $n$ vertices in a lower, and the remaining $n$ vertices in an upper row such that the $(n+i)$-th vertex is above the $i$-th vertex for $1 \leq i \leq n$. Now the $r$-th edge connects the vertices $i_{r}$ and $j_{r}$ in our graph, and and horizontal edges (i.e. edges which connect vertices which are in the same row) should be drawn slightly concave. Multiplication of a graph $a$ with a graph $b$ is given by putting $a$ on top of $b$, i.e., by identifying the $n$ lower vertices of $a$ with the $n$ upper vertices of $b$. The composite graph is again a basis graph, except that there may be cycles, i.e., components of the graph which are no longer connected with any upper or lower vertex. The product $a b$ is then defined to be the graph obtained by removing all cycles from the composite graph, multiplied by $x^{c}$, where $c$ is the number of cycles. As an instructive example, let $e$ denote the graph in $\mathcal{C}_{2}$ with horizontal upper and lower edges. Then putting two such graphs on top of each other produces a cycle (thanks to the concave drawing) between two horizontal edges and one obtains $e^{2}=x e$ (see e.g. W3, p. 181). 
Observe that $\mathcal{C}_{n}$ contains the group algebra of the symmetric group $S_{n}$ as a subalgebra; it is spanned by graphs which connects the $i$-th lower vertex to an upper vertex, say the $\pi(i)$-th vertex, for $i=1,2, \ldots n$, where $\pi \in S_{n}$; here we label both the lower and the upper vertices by the numbers 1 through $n$. We will call such graphs permutation graphs. It is not hard to see that $\mathcal{C}_{n}$ is generated by $S_{n}$ and $e_{1}=e \otimes 1_{n-2}$.

Moreover, we define for graphs $a \in \mathcal{C}_{n}, b \in \mathcal{C}_{m}$ a new graph $a \otimes b \in \mathcal{C}_{n+m}$ by putting the graph $b$ to the right of the graph $a$. Then $a^{\otimes k}$ is equal to $a \otimes a \otimes \ldots \otimes a$ ( $k$ times) and the identity $1_{k}$ for $\mathcal{C}_{k}$ is given by the graph with $k$ vertical edges.

Obviously, the definitions above go through if we replace $\mathbb{Z}[x]$ by any polynomial ring $R[x]$ for a unital abelian ring $R$ or by any quotient field $F(x)$ for $F$ a field. We will only consider characteristic 0 in this paper. Similarly, if $N \in R$ for a unital abelian ring, we define the algebra $\mathcal{C}_{n}(N)$ over $R$ via the same graphs by substituting $x=N$ in the multiplication. We denote by $\mathcal{C}_{n}(N)$ the $\mathbb{Q}$ algebra spanned by the basis graphs, with $x=N \in \mathbb{Q}$.

2.3. Centralizers. We have already seen how a basis graph is related to a map in (2.1). Combining this with the canonical isomorphism between $\left(V^{*}\right)^{\otimes 2 n}$ and $\operatorname{End}\left(V^{\otimes n}\right)$, we obtain for each basis graph a map in $\operatorname{End}_{O(N)}\left(V^{\otimes n}\right)$. For example, for $n=2$ the three maps mentioned in Section 2.1 correspond to the identity map, the permutation map $v_{1} \otimes v_{2} \mapsto v_{2} \otimes v_{1}$ and to the map

$$
\Phi(e): v \otimes w \mapsto(v, w) \sum_{i=1}^{N} f_{i} \otimes f_{i},
$$

where $\left(f_{i}\right)$ is an orthonormal basis of $V$. It is easy to check that $\Phi(e)^{2}=N \Phi(e)$. We can now formulate Brauer's result as follows:

Theorem 2.1. There exists a surjective homomorphism $\Phi$ of the algebra $\mathcal{C}_{n}(N)$, defined over $\mathbb{C}$ onto End ${ }_{O(N)}\left(V^{\otimes n}\right)$. It can be explicitly described by mapping permutation graphs to the corresponding permutation of factors in $V^{\otimes n}$, and by mapping $e_{1}$ to $\Phi(e) \otimes 1_{n-2}$, where $\Phi(e)$ is as in (2.2) and $1_{n-2}$ is the identity map of $V^{\otimes n-2}$. Moreover, this representation is faithful if $N>n$.

2.4. Symplectic case. The same strategy also works for describing $\operatorname{End}_{S p(N)}\left(V^{\otimes n}\right)$, where $V=\mathbb{C}^{N}$ is the vector representation of the symplectic group $S p(N)$, with $N$ even. Now, let $($,$) be a nondegenerate symplectic bilinear form on V$, and let $\left(f_{i}\right),\left(g_{i}\right)$ be dual bases with respect to $($,$) . Then we define \Phi(e) \in \operatorname{End}\left(V^{\otimes 2}\right)$ by $\Phi(e)(v \otimes w)=(v, w) \sum_{i} f_{i} \otimes g_{i}$.

Theorem 2.2. There exists a surjective homomorphism $\Phi$ of the algebra $\mathcal{C}_{n}(-N)$, defined over $\mathbb{C}$ onto End $\operatorname{En}_{S p(N)}\left(V^{\otimes n}\right)$. It can be explicitly described by mapping permutation graphs to the corresponding signed permutation of factors in $V^{\otimes n}$, and by mapping $e_{1}$ to $-\Phi(e) \otimes 1_{n-2}$, where $\Phi(e)$ is as defined in this subsection. Moreover, this representation is faithful if $|N|>n$.

Remark 2.3. There also exists a symplectic Brauer algebra which maps surjectively onto $\operatorname{End}_{S p(N)}\left(V^{\otimes n}\right)$, and for which the homomorphism in the last theorem is a little more natural. We will only use the orthogonal Brauer algebra, although this leads to some minor complications in notation later. 
2.5. $q$-Version of Brauer algebra. We also need the complex algebra $\hat{\mathcal{C}}_{n}(r, q)$ depending on complex parameters $r$ and $q$. We denote by $\hat{\mathcal{C}}_{n}$ the corresponding algebra, defined over a field of rational functions where $r$ and $q$ are considered variables. The algebra $\hat{\mathcal{C}}_{n}(r, q)$ is defined via generators $T_{1}, T_{2} \ldots T_{n-1}$, which satisfy the braid relations $T_{i} T_{i+1} T_{i}=T_{i+1} T_{i} T_{i+1}$ and $T_{i} T_{j}=T_{j} T_{i}$ if $|i-j|>1$ as well as the relations

(R1) $E_{i} T_{i}=r^{-1} E_{i}$,

(R2) $E_{i} T_{i-1}^{ \pm 1} E_{i}=r^{ \pm 1} E_{i}$, where $E_{i}$ is defined by the equation

$$
\left(q-q^{-1}\right)\left(1-E_{i}\right)=T_{i}-T_{i}^{-1} .
$$

The algebras $\hat{\mathcal{C}}_{n}(r, q)$ have a trace functional $t r$. To define it, it will be convenient to use the notation $x=\frac{r-r^{-1}}{q-q^{-1}}+1$. Then $\operatorname{tr}$ can be defined inductively by $\operatorname{tr}(1)=1$, $\operatorname{tr}\left(T_{i}\right)=r / x, \operatorname{tr}\left(E_{i}\right)=1 / x$ and $\operatorname{tr}(a \chi b)=\operatorname{tr}(a b) \operatorname{tr}(\chi)$ for $a, b \in \hat{\mathcal{C}}_{n}$ and $\chi \in$ $\left\{1, T_{n}, E_{n}\right\}$. The algebra $\hat{\mathcal{C}}_{n}\left(q^{N-1}, q\right)$ plays the same role for the Drinfeld-Jimbo quantum group $U_{q} s o_{N}$ as the Brauer algebra $\mathcal{C}_{n}(N)$ plays for the orthogonal group $O(N)$. Moreover, analogues of the graph basis for Brauer algebras have been defined in GH] (see also references there). In this set-up, one obtains the algebra $\mathcal{C}_{n}(N)$ as a $\operatorname{limit}_{\lim } \operatorname{li}_{q} \hat{\mathcal{C}}_{n}\left(q^{N-1}, q\right)$.

2.6. Algebraic structure. As usual, a Young diagram $\lambda=\left(\lambda_{i}\right)$ is an array of boxes, with $\lambda_{i}$ boxes in the $i$-th row. We will freely identify $\lambda$ with a vector in $\mathbb{Z}^{m}$ whose $i$-th coordinate is equal to $\lambda_{i}$ whenever $m$ is greater than the number of rows. Let $|\lambda|$ be the number of boxes of $\lambda$. We denote by $\lambda^{\prime}$ the Young diagram with rows and columns interchanged. In particular, $\lambda_{i}^{\prime}$ is the number of boxes in the $i$-th column.

Structure of $\mathcal{C}_{n}$ and $\hat{\mathcal{C}}_{n}$. (a) The algebra $\mathcal{C}_{n}$ is a direct sum of full matrix algebras. Its simple components are labelled by the Young diagrams with $n, n-2, n-4, \ldots$, 1 , respectively, 0 boxes.

(b) Let $U_{n, \lambda}$ be a simple $\mathcal{C}_{n, \lambda}$ module. The decomposition of $U_{n, \lambda}$ into simple $\mathcal{C}_{n-1}$ modules is given by

$$
U_{n, \lambda} \cong \bigoplus_{\mu} U_{n-1, \mu}
$$

where the summation goes over all Young diagrams $\mu$ which can be obtained by either taking away or, if $\lambda$ has less than $n$ boxes, by adding a box to $\lambda$. The labeling of simple components is uniquely determined by the restriction rule, except for a possible choice of replacing $\lambda$ by its transposed $\lambda^{\prime}$ simultaneously for all Young diagrams (see e.g. W1, Lemma 2.11). The dimension of $U_{n, \lambda}$ is determined by this formula inductively. There also exist explicit formulas for them; see e.g. [R].

(c) The analogous statements for semisimplicity and restriction rules also hold for the algebra $\hat{\mathcal{C}}_{n}$. Here we have the convention that the eigenprojection of $T_{1}$ corresponding to its eigenvalue $q$ is labeled by the Young diagram [2].

(d) There exists a similar theory for the integral versions of $\mathcal{C}_{n}(N)$ and $\hat{\mathcal{C}}_{n}(r, q)$ defined over suitable rings (see e.g. $\mathrm{GH}$ ] and the references therein). It was shown that both series of algebras are cellular in the sense of [GL; see [GL] and [Xi]. This means, in particular, that we can also find suitable bases for the modules $U_{n, \lambda}$ with respect to which the generators act via matrices with entries in those rings. Every simple module of $\mathcal{C}_{n}(N)$ and $\hat{\mathcal{C}}_{n}(r, q)$ appears as a quotient of a $U_{n, \lambda}$ with respect 
to the annihilator of a certain bilinear form, and also if the algebras themselves are not semisimple. This can happen for $\mathcal{C}_{n}(N)$ if $N$ is an integer or for $\hat{\mathcal{C}}_{n}(r, q)$ if $q$ is a root of unity and/or if $r= \pm q^{k}$ for some integer $k$; see [W2, W3.

(e) The labeling of simple components of $\mathcal{C}_{n}$ and $\mathcal{C}_{n}(N)$ via Young diagrams is chosen such that the idempotent $p_{\left[1^{2}\right]}$ corresponds to the antisymmetrization of $V^{\otimes 2}$ for $O(N)$ and to the symmetrization of $V^{\otimes 2}$ for $S p(|N|)$ under the map $\Phi$ in Theorems 2.1 and 2.2. This entails that a minimal idempotent $p_{\lambda}$ with $\Phi\left(p_{\lambda}\right) \neq 0$ projects onto a simple $O(N)$-module labeled by $\lambda$, and onto a simple $S p(N)$-module with highest weight $\lambda^{\prime}$.

2.7. Special quotients. Let us first do the orthogonal case. Let $\hat{\mathcal{C}}_{n}(M, \ell)$ be the $\mathbb{C}$ algebra defined by the $T_{i}$ 's and $E_{i}$ 's as above with $q=e^{\pi i / \ell}$ and $r=q^{M-1}$. The following statements would hold as well if $q$ is replaced by any primitive $2 \ell$-th root of unity. We define $\overline{\mathcal{C}}_{n}(M, \ell)$ to be the quotient of $\hat{\mathcal{C}}_{n}(M, \ell)$ modulo the annihilator ideal $I_{n}(M, \ell)$ of $t r$, i.e., $I_{n}(M, \ell)$ consists of all the elements $a \in \hat{\mathcal{C}}_{n}(M, \ell)$ such that $\operatorname{tr}(a b)=0$ for all $b \in \hat{\mathcal{C}}_{n}(M, \ell)$. Then we have the following properties:

(a) We have $I_{n}(M, \ell)=I_{n+1}(M, \ell) \cap \hat{\mathcal{C}}_{n}(M, \ell)$, so we obtain well-defined embeddings $\overline{\mathcal{C}}_{n}(M, \ell) \subset \overline{\mathcal{C}}_{n+1}(M, \ell)$.

(b) The quotients $\overline{\mathcal{C}}_{n}(M, \ell)$ are semisimple and its simple components are labeled by Young diagrams $\lambda$ with $n, n-2, \ldots$ boxes satisfying $\lambda_{1}^{\prime}+\lambda_{2}^{\prime} \leq M$ and $\lambda_{1}+\lambda_{2} \leq$ $\ell+1-M$ for $M>0$.

(c) The restriction rule for simple $\overline{\mathcal{C}_{n}}(M, \ell)$ modules is the same as for the generic case, as described in the previous chapter, except that now only the diagrams satisfying the conditions in part (b) are allowed.

For the symplectic case, let $M<0$ be even. We define $\hat{\mathcal{C}}_{n}(M, \ell)$ and $\overline{\mathcal{C}}_{n}(M, \ell)$ as before with $q=e^{\pi i / 2 \ell}$ and $r=q^{M-1}$. Then statements (a) and (c) above also hold in this case, while for statement (b) now the Young diagrams have to satisfy the conditions $\lambda_{1} \leq|M| / 2$ and $\lambda_{1}^{\prime} \leq \ell-|M| / 2$.

Remark 2.4. It follows from the semisimplicity of representations that the multiplicity of a simple $O(N)$ module $V_{\lambda}$ labeled by the Young diagram $\lambda$ is equal to the dimension of a simple $\Phi\left(\mathcal{C}_{n, \lambda}\right)$-module $\tilde{W}_{n, \lambda}$, where $\Phi$ is the same as in (2.2). These dimensions can be calculated inductively by the restriction rule as in (2.3), but now where only admissible Young diagrams are allowed; admissible $O(M)$ and $S p(M)$ diagrams are as defined above with $\ell=\infty$, i.e., one of the two conditions above becomes void. Observe that this is equivalent to the tensor product rule for $V_{\lambda} \otimes V$; it is well known that this is a direct sum of simple modules $V_{\mu}$, where $\mu$ runs through all admissible diagrams which can be obtained by adding or removing a box to/from $\lambda$.

While the ring $\operatorname{Gr}(G)_{\ell}$ is not the representation ring of a group, we will see in the next section that it is the representation ring of a semisimple tensor category $\mathcal{F}=\mathcal{F}\left(O(N)_{\ell}\right)$. If $X_{\lambda}$ is the simple object in $\mathcal{F}$ labeled by $\lambda \in G r(G)_{\ell}$, and, for $G=O(N)$ or $G=S p(N), X=X_{[1]}$ is the object corresponding to the vector representation, we again get that $X_{\lambda} \otimes X \cong \bigoplus_{\mu} X_{\mu}$, where $\mu$ runs through all admissible diagrams (for $G r(G)_{\ell}$ ) which can be obtained by adding or removing a box to/from $\lambda$. It follows as before that $\operatorname{End}_{\mathcal{F}}\left(X^{\otimes n}\right) \cong \overline{\mathcal{C}}_{n}(N, \ell)$.

Lemma 2.5. Let $T_{i}(r, q)$ and $E_{i}(r, q)$ denote the generators of $\hat{\mathcal{C}}_{n}(r, q)$. 
(a) The map $T_{i}\left(-r^{-1}, q\right) \mapsto-T_{i}^{-1}(r, q)$ defines an isomorphism between $\hat{\mathcal{C}}_{n}\left(-r^{-1}, q\right)$ and $\hat{\mathcal{C}}_{n}(r, q)$ which maps the simple component $\hat{\mathcal{C}}_{n}(r, q)_{\lambda}$ to $\hat{\mathcal{C}}_{n}\left(-r^{-1}, q\right)_{\lambda^{\prime}}$.

(b) Let $q$ be a primitive $2 \ell$-th root of unity, and let $1<M<\ell$. Then the map in (a) also induces an isomorphism between $\overline{\mathcal{C}}_{n}(M, \ell)$ and $\overline{\mathcal{C}}_{n}(\ell+2-M, \ell)$. Moreover, these isomorphisms are compatible with the standard inclusions $\overline{\mathcal{C}}_{n}(M, \ell) \subset$ $\overline{\mathcal{C}}_{n+1}(M, \ell)$.

(c) Let $q$ be a primitive $4 \ell$-th root of unity, and let $-2 \ell<M<-1$, with $M$ even. Then the map in (a) also induces an isomorphism between $\overline{\mathcal{C}}_{n}(M, \ell)$ and $\overline{\mathcal{C}}_{n}(-2 \ell+|M|+2, \ell)$, which is again compatible with inclusions.

Proof. The isomorphism property is easy to check. It maps the eigenprojection of $T_{1}\left(-r^{-1}, q\right)$ for $q$ (the component of $\hat{\mathcal{C}}_{2}\left(-r^{-1}, q\right)$ labeled by [2]) to the eigenprojection of $T_{i}(r, q)$ of $-q^{-1}$, which is labeled by the Young diagram [1 $1^{2}$. By uniqueness of labeling of the simple components, this implies that the simple component $\hat{\mathcal{C}}_{n}\left(-r^{-1}, q\right)_{\lambda}$ is mapped to the simple component $\hat{\mathcal{C}}_{n}(r, q)_{\lambda^{\prime}}$. This shows (a).

Now observe that the map on $\hat{\mathcal{C}}_{n}\left(-r^{-1}, q\right)$ induced by the concatenation of our map with the Markov trace on $(\hat{\mathcal{C}}(r, q))$ coincides with the Markov trace on $\hat{\mathcal{C}}_{n}\left(-r^{-1}, q\right)$. Indeed, by the inductive definition of the trace, it suffices to check this for the generators. As $x(r, q)=x\left(-r^{-1}, q\right)$ (see definitions of $\left.(\hat{\mathcal{C}}(r, q))\right)$, it follows that $\operatorname{tr}\left(E_{i}(r, q)\right)=\operatorname{tr}\left(E_{i}\left(-r^{-1}, q\right)\right)$. Using relation $(D)$, one also sees that $\operatorname{tr}\left(T_{i}^{-1}(r, q)\right)=r^{-1} / x$. Hence also $\operatorname{tr}\left(T_{i}\left(-r^{-1}\right)\right)=\operatorname{tr}\left(-T_{i}^{-1}(r, q)\right)$, which proves our claim.

Now if $q$ is a primitive $2 \ell$-th root of unity, and $r=q^{M-1}$, we have $-r^{-1}=$ $q^{\ell} q^{1-M}=q^{\ell+1-M}$. It follows from the two previous paragraphs that we have a trace preserving isomorphism between $\hat{\mathcal{C}}_{n}(M, \ell)$ and $\hat{\mathcal{C}}_{n}(\ell+2-M, \ell)$. The claim follows from this and the definitions of $\overline{\mathcal{C}}_{n}(M, \ell)$ and $\overline{\mathcal{C}}_{n}(\ell+2-M, \ell)$. The proof for (c) goes similarly.

\section{GRothendieCK SEMIRINGS}

The main purpose of this section is to prove Proposition 1.8, which is a case of what is known as level-rank duality. This has been observed before in the physics and mathematics literature (see e.g. the discussions to Chapters 16 and 17 in [FMS and, e.g., the papers [BB] and [MPS]). We will give a fairly simple and elementary proof for the particular cases that we need, which is more or less the same approach as the one in the papers $[\mathrm{BB}$ ] and $\mathrm{MPS}$, which go back to results in W2 and [TW2]. It will hopefully make the translation between the various approaches to our setting as well as fixing notations easier. It should also explain the rings $\operatorname{Gr}(G)_{\ell}$ of the first section to some extent, and will put some of the other topics discussed later into a more conceptual setting.

3.1. Semirings from idempotents. Let $G=O(N)$ or $G=S p(N)$, and let $\Phi: \mathcal{C}_{n}( \pm N) \rightarrow \operatorname{End}_{G}\left(V^{\otimes n}\right)$ be the surjective homomorphism mentioned in Section 2.2. As an idempotent $p \in \mathcal{C}_{n}(N)$ corresponds to the subrepresentation $\Phi(p) V^{\otimes n}$, we can translate the tensor product structure of $\operatorname{Rep}(G)$ into the setting of the algebras $\mathcal{C}_{n}(N)$. More generally, this can also be done as well for the algebras $\mathcal{C}_{n}$ and $\hat{\mathcal{C}}_{n}$ which will lead to the definition of a formal Grothendieck semiring $G r(O(\infty))_{+}$. The following definitions and constructions have appeared before, 
e.g., in $K$-theory for $C^{*}$-algebras and the idempotent construction for categories, also sometimes referred to as the Karoubian.

We say that two idempotents $p$ and $q$ in an algebra $\mathcal{A}$ are conjugation equivalent if there exist elements $u$ and $v$ in $\mathcal{A}$ such that $p=u v$ and $q=v u$. More generally, we say that two idempotents $p \in \mathcal{C}_{n}$ and $q \in \mathcal{C}_{m}$ are equivalent if we can find nonnegative integers $n_{1}$ and $n_{2}$ such that $\left(\frac{1}{x} e\right)^{\otimes n_{1}} \otimes p$ and $\left(\frac{1}{x} e\right)^{\otimes n_{2}} \otimes q$ are conjugation equivalent in the algebra $\mathcal{C}_{n+2 n_{1}}=\mathcal{C}_{m+2 n_{2}}$. For a given idempotent $p \in \mathcal{C}_{n}$ we denote by $[p]$ its equivalence class. It is easy to check that for idempotents $p \in \mathcal{C}_{n}$ and $q \in \mathcal{C}_{m}$ we obtain an idempotent $p \otimes q \in \mathcal{C}_{n+m}$ which gives rise to a well-defined multiplication $[p][q]=[p \otimes q]$.

Addition is defined as follows: Assume $p$ and $q$ are idempotents in $\mathcal{C}_{n}$ such that $q$ is conjugation equivalent to an idempotent $q^{\prime} \in \mathcal{C}_{n}$ satisfying $p q^{\prime}=0=q^{\prime} p$. Then also $p+q^{\prime}$ is an idempotent, and we define $[p]+[q]=\left[p+q^{\prime}\right]$. It is easy to check that this is well defined. Moreover, it is known that for the fixed Young diagram $\lambda$ the dimension of the simple components $\mathcal{C}_{|\lambda|+2 k, \lambda}$ goes to infinity if $k \rightarrow \infty$ (see the formula in the last section (b)). Hence, by tensoring $p$ and $q$ by a projection $\left(\frac{1}{x} e\right)^{\otimes k}$ for suitably large $k$, if necessary, we can always assume the existence of a $q^{\prime}$ as above; indeed, it is easy to see that such a $q^{\prime}$ exists whenever the sum of the ranks of $p$ and $q$ in an irreducible representation is less than its dimension.

Let $p_{\lambda}$ and $p_{\mu}$ be minimal idempotents in the simple components of $\mathcal{C}_{n}$ and $\mathcal{C}_{m}$ labeled by $\lambda$ and $\mu$, respectively. We shall often just write $[\lambda]$ and $[\mu]$ for $\left[p_{\lambda}\right]$ and $\left[p_{\mu}\right]$. If we define $d_{\lambda \mu}^{\nu}$ to be the rank of the idempotent $p_{\lambda} \otimes p_{\mu}$ in the irreducible representation of $\mathcal{C}_{n+m}$ labeled by the Young diagram $\nu$, we obtain

$$
[\lambda][\mu]=\left[p_{\lambda} \otimes p_{\mu}\right]=\sum_{\nu} d_{\lambda \mu}^{\nu}[\nu] .
$$

We denote the semiring of equivalence classes of idempotents in $\bigcup \mathcal{C}_{n}$ with addition and multiplication as defined above by $\operatorname{Gr}(O(\infty))$. Observe that $\operatorname{Gr}(O(\infty))$ can also be defined as the free $\mathbb{N}$ module spanned by the equivalence classes of minimal idempotents in $\bigcup \mathcal{C}_{n}$, with multiplication defined by (3.1).

Both definitions work as well for the sequence of algebras $\mathcal{C}_{n}(N)$ and for the sequence of algebras $\hat{\mathcal{C}}_{n}$. In the latter case the tensor product $a \otimes b$ of elements $a \in \hat{\mathcal{C}}_{n}$ and $b \in \hat{\mathcal{C}}_{n}$ is defined by $a \otimes b=a \operatorname{sh}_{n}(b)$, where $\operatorname{sh}_{n}: \hat{\mathcal{C}}_{m} \rightarrow \hat{\mathcal{C}}_{n+m}$ is the homomorphism defined by $\operatorname{sh}_{n}\left(T_{i}\right)=T_{i+n}, \operatorname{sh}_{n}\left(E_{i}\right)=E_{i+n}$, for $i=1,2, \ldots, m-1$.

It should be clear that the same constructions also work for the sequences of algebras $\hat{\mathcal{C}}_{n}(r, q)$ and $\overline{\mathcal{C}}_{n}(n, \ell)$. Similarly, we can also define a semiring for the sequence of group algebras $\mathbb{C} S_{n}$ of the symmetric groups $S_{n}, n \in \mathbb{N}$. In view of Schur duality, a minimal idempotent $p_{\lambda} \in \mathbb{C} S_{n}$ corresponds to an irreducible representation $F^{\lambda}$ of $G l(N)$ for $N$ sufficiently large. The resulting semiring will be denoted by $\operatorname{Gr}(G l(\infty))$. We denote the structure coefficients in the semiring $G r(G l(\infty))$ by $c_{\lambda \mu}^{\nu}$; they give the multiplicity of the simple $G l(N)$-module $F^{\nu}$ in the tensor product $F^{\lambda} \otimes F^{\mu}$.

Definition 3.1. We define $G r(\mathcal{C}(N))_{+} \operatorname{resp} G r(\hat{\mathcal{C}}(r, q))_{+}$, respectively, $\operatorname{Gr}\left(\overline{\mathcal{C}}_{n}(N, \ell)\right)$ to be the semirings derived from the equivalence classes of idempotents of the algebras $\mathcal{C}_{n}(N)$, respectively $\hat{\mathcal{C}}_{n}(r, q)$, respectively $\overline{\mathcal{C}}_{n}(N, \ell) n \in \mathbb{N}, N \in \mathbb{Z}$, and $r, q \in \mathbb{C}$ and we denote by $\operatorname{Gr}(G l(\infty))_{\text {+ }}$ the semiring obtained from the group algebras $\mathbb{C} S_{n}$ of the symmetric groups $S_{n}, n \in \mathbb{N}$. The corresponding rings are denoted by the same symbols, without the + . 
Remark 3.2. It is possible to construct tensor categories from the algebras mentioned above; see [TW2], [BB], [TbW]. The semirings just defined would be the Grothendieck semirings of these tensor categories. We will discuss this and other constructions of such categories below.

\subsection{Algebraic properties.}

Lemma 3.3. Let $d_{\lambda \mu}^{\nu}(N)$ and $d_{\lambda \mu}^{\nu}$ be the structure coefficients for $\operatorname{Gr}(S O(N))_{+}$ and $\operatorname{Gr}(O(\infty))$, see Section 1.3 . Then we have

(a) $d_{\lambda \mu}^{\nu}(N)=d_{\lambda \mu}^{\nu}$ for $|N|$ sufficiently large,

(b) $\operatorname{Gr}(\mathcal{C})_{+} \cong \operatorname{Gr}(O(\infty))_{+}$.

Proof. Let $p_{\lambda}, p_{\mu}$ be minimal idempotents in $\mathcal{C}_{n}(N)$, defined over $\mathbb{Q}(x)$. If $N$ is not one of the finitely many poles in the coefficients of $p_{\lambda}, p_{\mu}$ (with respect to the graph basis), we also obtain well-defined idempotents $p_{\lambda}(N), p_{\mu}(N)$ in the $\mathbb{Q}$ algebra $\mathcal{C}_{n}(N)$. Obviously, both $p_{\lambda} \otimes p_{\mu}$ and $p_{\lambda}(N) \otimes p_{\mu}(N)$ act as idempotents with the same rank $d_{\lambda \mu}^{\nu}$ on the module $U_{n, \nu}$. On the other hand, for $N>n$, $\operatorname{End}_{O(N)}\left(V^{\otimes n}\right) \cong \mathcal{C}_{n}(N)$, and hence $d_{\lambda \mu}^{\nu}$ is equal to the multiplicity $d_{\lambda \mu}^{\nu}(N)$ of the simple $O(N)$-module $V_{\nu}$ in $V_{\lambda} \otimes V_{\mu}$. Finally, assuming $N$ big enough, we can identify the Young diagram $\lambda$ as well with the corresponding $S O(N)$ dominant weight. This proves both (a) and (b).

Lemma 3.4. Let $\lambda^{\prime}$ denote the transpose of the Young diagram $\lambda$.

(a) The map $[\lambda] \mapsto\left[\lambda^{\prime}\right]$ defines an automorphism of $\operatorname{Gr}(O(\infty))$.

(b) The map $[\lambda] \mapsto\left[\lambda^{\prime}\right]$ defines an isomorphism between $\operatorname{Gr}\left(\overline{\mathcal{C}}_{n}(N, \ell)\right)$ and $\operatorname{Gr}\left(\overline{\mathcal{C}}_{n}(\ell+2-N, \ell)\right)$

Proof. This follows from Lemma 2.5.

3.3. Ribbon tensor categories. We give here some very limited background on such tensor categories. The reader can find very good introductions to tensor categories suitable for our context in $[\mathrm{Ks}$ ] and $[\mathrm{Tu}]$.

In the following we assume $\mathcal{T}$ to be a monoidal rigid ribbon tensor category. This means that we have a functor $\otimes: \mathcal{T} \times \mathcal{T} \rightarrow \mathcal{T}$ satisfying certain associativity conditions (monoidal) and rigidity, i.e., every object has a dual object (in the sense of a dual representation for group representations). We also have a braiding which means that there exist for any pair of objects $X, Y$ in $\mathcal{T}$ a canonical morphism $c_{X, Y}: X \otimes Y \rightarrow Y \otimes X$ subject to several axioms. In particular, we obtain for any object $X$ a representation of Artin's braid group $B_{n}$ into $\operatorname{End}\left(X^{\otimes n}\right)$. Conversely, one can construct from suitable series of braid group representations a monoidal rigid ribbon tensor categories. This has been carried out for the algebras $\overline{\mathcal{C}}_{n}(N, \ell)$ in TW2. In particular, $\operatorname{Gr}(\overline{\mathcal{C}}(N, \ell))_{+}$is the Grothendieck semiring of this category.

A special class of ribbon categories are modular tensor categories. To explain it, we first remark that one can define for any object $X$ in a rigid ribbon category a trace functional $\operatorname{Tr}_{X}$ on $\operatorname{End}(X)$ (see e.g. [Ks] Ch. XIV, 4). In particular, one defines the categorical dimension $d(X)$ of an object $X$ by $d(X)=\operatorname{Tr}_{X}\left(1_{X}\right)$, where $1_{X}$ is the identity in $\operatorname{End}(X)$. If $X_{\lambda}, X_{\mu}$ are simple objects in our tensor category, we define the scalar $s_{\lambda \mu}=\operatorname{Tr}_{X_{\lambda} \otimes X_{\mu}}\left(c_{X_{\mu} \otimes X_{\lambda}} \circ c_{X_{\lambda} \otimes X_{\mu}}\right)$. A modular tensor category is defined to be a semisimple rigid ribbon tensor category with only finitely many simple objects up to isomorphism for which the matrix $S=\left(s_{\lambda \mu}\right)$ is invertible. Now for any simple object $X_{\mu}$ of a ribbon category $\mathcal{T}$ with nonzero dimension $d\left(X_{\mu}\right)$ 
one can show the following Verlinde formula (see e.g. [Tu], Th. 4.5.1): The map $\phi_{\mu}:\left[X_{\lambda}\right] \mapsto s_{\lambda \mu} / d\left(X_{\mu}\right)$ defines a multiplicative character of $\operatorname{Gr}(\mathcal{T})$. In particular, for a modular tensor category, we thus obtain a complete set of characters of the abelian ring $\operatorname{Gr}(\mathcal{T})$; indeed, the characters $\left(\phi_{\mu}\right)$ are linearly independent due to the invertibility of the $S$-matrix.

3.4. Examples. An important class of ribbon tensor categories is provided by the Drinfeld-Jimbo $q$-deformations $U_{q} \mathfrak{g}$ of the universal enveloping algebra of a semisimple Lie algebra $\mathfrak{g}$. For generic $q$ (i.e. $q$ not a root of unity except for $q= \pm 1$ ) the category of finite dimensional representations of $U_{q} \mathfrak{g}$ is semisimple. Moreover, its simple representations are labeled by the dominant weights of $\mathfrak{g}$. This provides an isomorphism between the Grothendieck semirings of $\operatorname{Rep}(\mathfrak{g})$ and of $\operatorname{Rep}\left(U_{q} \mathfrak{g}\right)$, where Rep refers to finite dimensional representations.

It is more complicated to construct modular tensor categories. We will rely here on constructions using tilting modules of quantum groups at roots of unity (see $\mathrm{A}$, $[\mathrm{AP}$ ). There are also more elementary constructions (see [TW2, [B], [BB]) which would at least cover some of the cases needed here; but they are not as general. Additional constructions can be found in $[\mathrm{KL}$ and $\mathrm{Wa}$. We have the following theorem:

Theorem 3.5. For each connected and simply connected Lie group $G$ and positive integer $\ell$ exists a modular tensor category $\mathcal{F}=\mathcal{F}\left(G_{\ell}\right)$ whose Grothendieck semiring is isomorphic to $\operatorname{Gr}(G)_{\ell,+}$ as in Section 1.1 .

Proof. This is essentially contained in $\mathrm{A}$, $\mathrm{AP}$. The authors construct a braided tensor category $\mathcal{F}$ as a quotient of the category of tilting modules of a quantum group $U_{q} \mathfrak{g}$ for $q$ a root of unity. Its simple objects $T_{\lambda}$ (up to isomorphism) are labeled by the same index set as the basis elements of $\operatorname{Gr}(G)_{\ell}$, where $G$ is the simply connected Lie group corresponding to $\mathfrak{g}$ and $\ell$ is determined by the degree of the root of unity. In particular, the entries of the $S$-matrix can be calculated using the theory of $R$-matrices (see e.g. [TW1, Lemma 3.5.1). If $q=e^{\pi i / \ell}$, one can explicitly calculate that $s_{\lambda \mu}=\chi^{\lambda}((\mu+\rho) / \ell) d\left(X_{\mu}\right)$, where $\chi^{\lambda}$ is given by the Weyl character formula, and $d\left(X_{\mu}\right)=d(\mu)$ as in Section 1.1 in particular, $\left(s_{\lambda \mu}\right)$ is invertible. Hence the characters $\phi_{\mu}$ mentioned in this section coincide with the ones in Proposition 1.1, and the Grothendieck semiring of $\mathcal{F}$ is isomorphic to $\operatorname{Gr}(G)_{\ell}$. In particular, the subset $\operatorname{Gr}(G)_{\ell,+} \subset \operatorname{Gr}(G)_{\ell}$ (see Remark 1.2) is closed under multiplication and hence forms a semiring.

3.5. Identification with $\operatorname{Gr}\left(\overline{\mathbb{C}}_{n}(N, \ell)\right)$. As outlined in the beginning of this section, we can identify the ring $\operatorname{Gr}(O(N))$ with $\operatorname{Gr}(\Phi(\mathcal{C}(N)))$, as $\Phi\left(\mathcal{C}_{n}(N)\right) \cong$ $\operatorname{End}_{O(N)}\left(V^{\otimes n}\right)$. We are going to prove similarly that the semiring $\operatorname{Gr}\left(\overline{\mathcal{C}}_{n}(N, \ell)\right)_{+}$ is isomorphic to the Grothendieck semiring of a suitable fusion category. The only slight subtlety comes from the fact that $\operatorname{Gr}\left(\overline{\mathcal{C}}_{n}(N, \ell)\right)$ would correspond to a fusion category in connection with $O(N)$, while the ones obtained from quantum groups would correspond to $S O(N)$.

Proposition 3.6. (a) $\operatorname{Gr}(\overline{\mathcal{C}}(-N, 2 \ell))_{+} \cong G r(S p(N))_{\ell,+}$ for $N$ even.

(b) $\operatorname{Gr}(\overline{\mathcal{C}}(N, \ell))_{+} \cong G r(O(N))_{\ell,+}$

Proof. We will check that the stated isomorphisms are given by associating the equivalence class $\left[p_{\lambda}\right]$, with $p_{\lambda}$ a minimal idempotent in $\hat{\mathcal{C}}_{n, \lambda}\left(q^{ \pm N-1}, q\right)$, to the element $\lambda$ in $\operatorname{Gr}(O(N))_{\ell,+}$, respectively, $\operatorname{Gr}(S p(N))_{\ell,+}$. Let $\mathcal{F}$ be one of the fusion 
categories related to an orthogonal or symplectic group, and let $X=X_{[1]}$ be a simple object in $\mathcal{F}$ whose isomorphism class is labeled by the Young diagram [1]. Then it is well known that the representation of the braid group $B_{n}$ derived from the braiding morphisms $c_{X, X}$ is a quotient of $\hat{\mathcal{C}}\left(q^{N-1}, q\right.$ ) (for $G=O(N)$ ) or of $\hat{\mathcal{C}}\left(q^{-1-N}, q\right)$ (for $\left.G=S p(N)\right)$; see e.g. [Tu1, W2] or [TbW]. Moreover, the pullback of the categorical trace $T r_{X \otimes n}$ on $\operatorname{End}\left(X^{\otimes n}\right)$ induces the Markov trace of $\hat{\mathcal{C}}_{n}$, up to a multiple (see e.g. [TbW], Cor 5.3). Hence the quotient $\overline{\mathcal{C}}_{n}\left(q^{N-1}, q\right)=$ $\hat{\mathcal{C}}_{n}\left(q^{N-1}, q\right) / \operatorname{Ann}(t r)$ is also a quotient in the image of $B_{n}$ in $\operatorname{End}\left(X^{\otimes n}\right)$. Moreover, if we choose for the required $2 \ell$-th (orthogonal case) or $4 \ell$-th (symplectic case) root of unity $q=e^{\pi i / \ell}$ or $q=e^{\pi i / 2 \ell}$, respectively, $\operatorname{Tr}_{X} \otimes n$ induces an inner product on $\operatorname{End}\left(X^{\otimes n}\right)$ (see W5]). Hence the image of $\operatorname{Ann}(\operatorname{tr})$ in $\operatorname{End}\left(X^{\otimes n}\right)$ is 0 , and the aforementioned quotient is a subalgebra in $\operatorname{End}\left(X^{\otimes n}\right)$.

Comparing the tensor product rules in $\mathcal{F}$ with the embeddings of $\overline{\mathcal{C}}_{n} \subset \overline{\mathcal{C}}_{n+1}$, one checks by induction on $n$ that for $G=S p(N)$ and for $G=O(N)$ with $N$ odd, we have $\operatorname{End}_{\mathcal{F}}\left(X^{\otimes n}\right) \cong \overline{\mathcal{C}}_{n}\left(q^{-N-1}, q\right)($ for $S p(N))$, and $\cong \overline{\mathcal{C}}_{n}\left(q^{N-1}, q\right)($ for $O(N))$; see Sections 2.6, 2.7 and Remark 2.4. Moreover, these isomorphisms are compatible with the tensor product operation. Hence we get the desired isomorphism of Grothendieck semirings.

For the even-dimensional case, we will show that $\overline{\mathcal{C}}_{n}\left(q^{N-1}, q\right)$ is isomorphic to a subalgebra of $\operatorname{End}\left(X^{\otimes n}\right)$ for which the inclusion is compatible with the restriction rules from $\operatorname{Gr}(O(N))_{\ell}$ to $\operatorname{Gr}(S O(N))_{\ell}$. It follows from Brauer's results that $\Phi\left(\tilde{p}_{\lambda}\right) V^{\otimes n}$ decomposes as an $S O(N)$-module according to the restriction rules, as, e.g., stated in Section 1.6, here $\tilde{p}_{\lambda}$ is a minimal idempotent in $\mathcal{C}_{n, \lambda}(N)$. We claim that it suffices to find, for given $\lambda \in D_{\ell}$, an idempotent $p_{\lambda}=p_{\lambda}(q) \in \hat{\mathcal{C}}_{n, \lambda}\left(q^{N-1}, q\right)$ such that $p_{\lambda}(1)=\tilde{p}_{\lambda}$ and which is well defined at $q=e^{\pi i / \ell}$. To see this, consider the explicit representation of $\hat{\mathcal{C}}_{n, \lambda}\left(q^{N-1}, q\right)$ into $\operatorname{End}\left(V^{\otimes n}\right)$ derived from Jimbo's $R$-matrices; see [W2, Section 5, or [Tu1]. Here the basis vectors of $V=\mathcal{C}^{N}$ are weight vectors of the quantum group $U_{q} \mathfrak{s o}$ for any $q$. As the image of $\hat{\mathcal{C}}_{n, \lambda}\left(q^{N-1}, q\right)$ commutes with the action of $U_{q} \mathfrak{s o} o_{N}$, each weight space of $V^{\otimes n}$ is invariant under $\hat{\mathcal{C}}_{n, \lambda}\left(q^{N-1}, q\right)$ for any $q$. In particular, the $U_{q} \mathfrak{s} o_{N}$ character of $p_{\lambda} V^{\otimes n}$ does not depend on $q$, and the restriction rule also holds for $p_{\lambda}\left(e^{\pi i / \ell}\right)$. As $G r(O(N))_{\ell}$ was defined from $\operatorname{Gr}(S O(N))_{\ell}$ via these restriction rules, the claim would follow.

Inductive formulas for certain minimal idempotents, called path idempotents, were defined in [RW], using prior work in [W1] for Hecke algebras; see also [LR] for a similar approach. The formulas are written explicitly, involving quantities such as hook lengths in Young diagrams. One can check that they are well defined for paths only involving diagrams in the labeling set of $\operatorname{Gr}(O(N))_{\ell}$ for $q=e^{\pi i / \ell}$, and that one can get a partition of unity in $\overline{\mathcal{C}}_{n}\left(q^{N-1}, q\right)$ in terms of such idempotents. This is fairly straightforward, using the explicit formulas in RW. This finishes the proof.

Proof of Proposition 1.8. In the orthogonal case, it follows from Proposition 3.6 that $\operatorname{Gr}(O(N))_{\ell,+} \cong \operatorname{Gr} \overline{\mathcal{C}}(N, \ell)_{+}$. Hence $\operatorname{Gr}(O(N))_{\ell,+} \cong G r(O(\ell+2-N))_{\ell,+}$, by Lemma 3.4. The proof is the same in the symplectic case.

\section{Restriction COEFFicients}

Let $p_{\lambda}$ be a minimal idempotent in $\mathbb{C} S_{n}$. Then it can be written as a sum of mutually commuting minimal idempotents in $\mathcal{C}_{n}$. Let $b_{\mu}^{\lambda}$ be the number of those 
idempotents which are in $\mathcal{C}_{n, \mu}$; equivalently, $b_{\mu}^{\lambda}$ is the trace of $p_{\lambda}$ in an irreducible $\mathcal{C}_{n, \mu}$-module. Again, these coefficients depend on whether we take the orthogonal or symplectic Brauer algebra, which will be made precise below.

The connection of these coefficients with representation theory of Lie groups is easily established as follows. Let $V$ be an $N$-dimensional vector space with $N>n$. By Schur duality, $F^{\lambda}=p_{\lambda} V^{\otimes n}$ is an irreducible $G l(N)$-module. As $N>n$, we obtain a faithful representation of the Brauer algebra $\mathcal{C}_{n}(N)$ on $V^{\otimes n}$ such that its image is isomorphic to $\operatorname{End}_{G}\left(V^{\otimes n}\right)$ for $G=O(N)$ or $G=S p(N)$. Hence it follows that the coefficient $b_{\mu}^{\lambda}$ determines the multiplicity of the simple $G$-module $V_{\mu}$ in $F^{\lambda}$. If $N \leq n$, this multiplicity will be denoted by $b_{\mu}^{\lambda}(G)$. Formulas for the coefficients $b_{\mu}^{\lambda}$ and $b_{\mu}^{\lambda}(G)$ already appeared in $\mathrm{Wy}$ (see Theorems $7.8 \mathrm{~F}$ and $7.9 \mathrm{C}$ ). An explicit formula for $b_{\mu}^{\lambda}$ was computed by Littlewood [Li] as follows, where part (c) is a simple consequence of parts (a) and (b) as well as Lemma 3.4. See also KT, Section 1.5 for a more detailed description of this result and its proof, and EW] whose general proof also includes a derivation of the classical case.

Theorem 4.1 (Littlewood). (a) In the orthogonal case, we have $b_{\mu}^{\lambda}=\sum_{\nu} c_{\mu \nu}^{\lambda}$, where the summation goes over all Young diagrams which have an even number of boxes in each row, and $c_{\mu \nu}^{\lambda}$ is the multiplicity of the simple $G l(N)$-module $F^{\nu}$ in $F^{\lambda} \otimes F^{\mu}$. In particular, $b_{\mu}^{\lambda}=0$ unless $\mu \subset \lambda$, and $b_{\lambda}^{\lambda}=1$.

(b) In the symplectic case, we have $b_{\mu}^{\lambda}=\sum_{\nu} c_{\mu \nu}^{\lambda}$, where the summation goes over all Young diagrams which have an even number of boxes in each column, and $c_{\mu \nu}^{\lambda}$ is as in (a).

(c) The orthogonal and symplectic restriction coefficients are related by $b_{\mu}^{\lambda}(O)=$ $b_{\mu^{\prime}}^{\lambda^{\prime}}(S p)$.

Recall that for $G=O(N)$ or $S p(N)$ we defined a labeling set $D(G)$ as a certain subset of the set of Young diagrams (see Sections 1.5 and 1.6). Recall that we have defined an action of a reflection group $W$ on Young diagrams for which the closure of $D(G)$ is a fundamental domain; see Sections 1.4 and 1.5. The corresponding sets $D(G)^{\prime}=\left\{\lambda^{\prime}, \lambda \in D(G)\right\}$ are the usual labeling sets of irreducible representations of $G$; see Theorem 1.9. Let $b_{\mu}^{\lambda}$ be the multiplicity of the simple $G$-module $V_{\mu}$ in the simple $G l(N)$-module $F^{\lambda}$. We can now express the restriction coefficients $b_{\mu}^{\lambda}(G)$ as follows (see also [EW], [KT], [Ki], [Su]).

Theorem 4.2. With notation as above, the restriction multiplicity $b_{\mu}^{\lambda}(G)$ is given by

$$
b_{\mu}^{\lambda}(G)=\sum_{w \in W} \varepsilon(w) b_{w^{\prime} \cdot \mu}^{\lambda} .
$$

Proof. Let $p_{\lambda} \in \mathbb{C} S_{n}$ be a minimal idempotent, and let $\mathcal{I}(G)$ be the ideal in $G r(O(\infty))$ such that $G r(G) \cong G r(O(\infty)) / I(G)$. Then we have

$$
\left[p_{\lambda}\right]=\sum_{\mu} b_{\mu}^{\lambda}[\mu] \equiv \sum_{\mu \in D(G)^{\prime}}\left(\sum_{w} \varepsilon(w) b_{w^{\prime} . \mu}^{\lambda}\right)[\mu] \bmod \mathcal{I}(G),
$$

by Theorem 1.9

Remark 4.3. It is tempting to consider the question whether there exist analogs of restriction coefficients for fusion categories. This does not seem to be possible with the categories we have considered here, e.g., there is no inclusion of quantum 
groups $U_{q} \mathfrak{s} o_{N} \subset U_{q} \mathfrak{s} l_{N}$. However, it is expected that one can extend fusion categories of type $A$ by additional objects corresponding to representations of a twisted Kac-Moody algebra. Tensoring a twisted with an untwisted representation would formally correspond to a restriction-induction process. In one of the examples, the multiplicities would be modifications of restriction coefficients from $G l(N)$ to $O(N)$, which, for given diagrams and large enough $\ell$ would be equal to the classical coefficients. We plan to study these questions in connection with subfactors of von Neumann factors.

\section{Structure of Brauer algebra and generalizations}

5.1. Introduction. When the first version of this paper was written and submitted, it was still an unsolved problem of how to determine the structure of Brauer algebras in the case when they are not semisimple. Our work so far, in combination with the work [So2] on tilting modules, suggested a conjecture what the solution should be, at least as far as determining the dimensions of the simple modules was concerned. This conjecture was also obtained, independently, in [CdVM and, more recently, a proof has been posted in $\mathrm{Mt}$. Moreover, the paper [ $\mathrm{Hu}$ has appeared in the meantime from which one can determine, in connection with So2, the structure of $\hat{\mathcal{C}}_{n}\left(q^{-N-1}, q\right)$ with $N$ even (the symplectic case) in many cases. We extend this to the generic case (i.e. for all but finitely many $q$ ) along the approach outlined in the earlier version. This might also give a more conceptual understanding of the situation in the classic Brauer algebra case.

5.2. Tilting modules and Kazhdan-Lusztig polynomials. We will need to review some basic facts of Kazhdan-Lustig polynomials. See $\mathrm{Hm}$ and, in particular, So1 for introductions to this subject. If $W$ is a reflection group, then Kazhdan-Lusztig polynomials $P_{v, w}$ are defined for any pair $w, v$ of elements in $W$ via induction on the length of $w$. In particular, $P_{v, w} \neq 0$ only if $v \leq w$ in Bruhat order. Similarly, if $U \subset W$ is a parabolic subgroup, one can define parabolic Kazhdan-Lusztig polynomials $P_{\bar{v}, \bar{w}}$ for the left cosets $W / U$. We will need this for the inclusion $W \subset W^{(l)}$, where $W$ is a Weyl group and $W^{(l)}$ is an affine reflection group; see Section [1.1, In this case, there exists a well-known geometric model for the left cosets $W^{(l)} / W$ in terms of alcoves in the dominant Weyl chamber. In particular, the parabolic Kazhdan-Lusztig polynomials can be calculated via paths from the fundamental alcove to the given one; see e.g. $\mathrm{GW}$ ] for a review and for further references. We will need the following definition, with notation as in Section 1.1 .

Definition 5.1. Let $\lambda, \mu$ be dominant weights such that $w \cdot \lambda \neq \lambda$ for any nontrivial $w \in W^{(l)}$ Assume that there exists $w \in W^{(l)}$ such that $\nu=w^{-1} . \lambda \in D_{\ell}$. Then we define $a_{\mu}^{\lambda}=P_{\bar{v}, \bar{w}}(1)$ if $v \cdot \nu=\mu$, and $a_{\mu}^{\lambda}=0$ if $\nu$ is not in the $W^{(l)}$-orbit of $\mu$.

To distinguish between the various cases, we will write $a_{\mu}^{\lambda}(M, \ell)$ for $G=S O(M)$ (where we assume $M$ to be odd) and $a_{\mu}^{\lambda}(-M, \ell)$ for $G=S p(M)$, with $M$ even. Observe that we also have actions of $S_{\infty} \subset W=W\left(D_{\infty}\right)$, (or of $W=W\left(B_{\infty}\right)$ in the symplectic case) on infinite sequences as defined in Section 1.4. We then define similarly the quantity $a_{\mu}^{\lambda}( \pm N)$ for Young diagrams $\lambda, \mu$ via parabolic KazhdanLusztig polynomials for $S_{\infty} \subset W$ with the action $w \cdot \lambda$ defined in Section 1.4 here $a_{\mu}^{\lambda}(-N)$ is defined for the symplectic case $G=S p(N)$; see Section 1.5. We now 
have the following simple lemma, which can be considered a refinement of Lemma 1.4 .

Lemma 5.2. We have $a_{\mu}^{\lambda}(M, N+M-2)=a_{\mu}^{\lambda}(N)$ and $a_{\mu}^{\lambda}(-M,(N+M+2) / 2)=$ $a_{\mu}^{\lambda}(-N)$ independent of $M$ provided it is sufficiently large.

Proof. We assume that $\lambda$ and $\mu$ are in the same $W$ orbit; otherwise there is nothing to show. It follows from the discussion in Section 1.4 and, in particular, the algorithm in the proof of Lemma 1.4 that for large enough $m=M / 2$, the subgroup of $W^{(l)}(m)$ generated by the reflections needed to map $\lambda$ and $\mu$ into $D(N)$ is independent of $m$ and can be viewed as a subgroup of $W=W\left(D_{\infty}\right)$. Hence the corresponding Kazhdan-Lusztig polynomial is independent of $m$, and coincides with the one calculated within $W$. The same argument works in the symplectic case.

Tilting modules were defined for quantum groups by H.H. Andersen A and before that, for algebraic groups, by Donkin. A tilting module has a filtration in terms of Weyl modules; this implies, in particular, that its character is a sum of ordinary simple characters. More precisely, it has been shown by Soergel in [So2] that the character $\chi_{T}^{\lambda}$ of an indecomposable tilting module $T_{\lambda}$ of the quantum group $U_{q} \mathfrak{g}$ with highest weight $\lambda$ can be written as

$$
\chi_{T}^{\lambda}=\sum_{\mu} a_{\mu}^{\lambda} \chi^{\mu}
$$

where $\chi^{\mu}$ is the character of the simple $\mathfrak{g}$-module with highest weight $\mu$ and $a_{\mu}^{\lambda}$ is as in Definition [5.1] here $\ell$ is related to the degree of the root of unity $q$ in $U_{q} \mathfrak{g}$ as indicated in our examples. We also note here that the vector representation $V$ of $U_{q} \mathfrak{g}$, with $\mathfrak{g}$ of classical Lie type, is a tilting module, and so is any tensor power $V^{\otimes n}$ and any direct summand of $V^{\otimes n}$. Finally, any tilting module is a direct sum of indecomposable tilting modules, and there exists exactly one indecomposable tilting module $T_{\lambda}$ with highest weight $\lambda$ for each dominant weight $\lambda$.

5.3. $q$-Brauer algebras. As already stated before, the algebras $\hat{\mathcal{C}}_{n}\left(q^{-N-1}, q\right)$ map surjectively onto the algebras $\operatorname{End}_{\mathbf{U}}\left(V^{\otimes n}\right)$, where $\mathbf{U}=U_{q \mathfrak{s}} p_{N}, N$ even, for $q$ not a root of unity. Moreover, for $N>n$, this representation is faithful (see Theorems 2.1 and 2.2 which carry over to the $q$-deformations). Recall that for $S p(N)$ we associate to the dominant weight $\lambda$ the Young diagram $\lambda^{\prime}$, which has $\lambda_{i}$ boxes in its $i$-th column, for labeling the corresponding $\hat{\mathcal{C}}_{n}\left(q^{-N-1}, q\right)$ module. It is shown in $\mathrm{Hu}$ that this is also true at roots of unity in context of tilting modules of $U_{q} \mathfrak{s} p_{N}$. So if $T_{\lambda} \subset V^{\otimes n}$ is an indecomposable tilting module with highest weight $\lambda$, we can find a minimal projection $p_{\lambda^{\prime}} \in \hat{\mathcal{C}}_{n}\left(q^{-N-1}, q\right)$ such that $T_{\lambda}=p_{\lambda^{\prime}} V^{\otimes n}$. Moreover, the equivalence classes of minimal idempotents in $\hat{\mathcal{C}}_{n}\left(q^{-N-1}, q\right)$ are in 1-1 correspondence to indecomposable tilting modules (again, any minimal idempotent in $\hat{\mathcal{C}}_{n}\left(q^{-N-1}, q\right)$ projects onto a direct summand of the tilting module $V^{\otimes n}$, which hence is itself an indecomposable tilting module). We have the following simple consequence of the theorems of Soergel and Hu:

Theorem 5.3. Assume that $M>n$ and $M$ even. Let $d_{n, \lambda}(-M, \ell)$ be the dimension of a simple $\hat{\mathcal{C}}_{n, \lambda}\left(q^{-M-1}, q\right)$-module, with $q$ a primitive $4 \ell$-th root of unity, and let 
$d_{n, \mu}$ be the dimension of $U_{n, \mu}$; see Section 2.6. Then we have

$$
\sum_{\lambda} d_{n, \lambda^{\prime}}(-M, \ell) a_{\mu}^{\lambda}(-M, \ell)=d_{n, \mu^{\prime}}
$$

These equations completely determine the dimensions $d_{n, \lambda}(-M, \ell)$ of all simple $\hat{\mathcal{C}}_{n, \lambda}\left(q^{-M-1}, q\right)$ modules.

Proof. Decomposing $V^{\otimes n}$ into a direct sum of simple $S p(M)$-modules, and into a direct sum of indecomposable tilting modules, and comparing characters, with our labeling conventions, we get

$$
\chi_{V \otimes n}=\sum_{\mu} d_{n, \mu^{\prime}} \chi^{\mu}=\sum_{\lambda} d_{n, \lambda^{\prime}}(-M, \ell) \chi_{T}^{\lambda} .
$$

The claim now follows from expanding $\chi_{T}^{\lambda}$ in ordinary characters; see (5.1), and comparing the coefficients of $\chi^{\mu}$ on both sides. Finally, it is well known that $a_{\mu}^{\lambda}(-M, \ell)$ is nonzero only if $\mu \leq \lambda$ in Bruhat order. Hence we get a triangular system of equations for the dimensions $d_{n, \lambda}(-M, \ell)$ in terms of the generic dimensions $d_{n, \lambda}$, which can be computed inductively; see Section 2.6.

Remark 5.4. 1. The condition $n<M$ is necessary to get a faithful representation of $\hat{\mathcal{C}}_{n}\left(q^{-M-1}, q\right)$ in $\operatorname{End}\left(V^{\otimes n}\right)($ see $[\mathrm{Hu}])$.

2. The matrix $\left(a_{\mu^{\prime}}^{\lambda^{\prime}}(-M, \ell)\right)$ is usually called the decomposition matrix for $\hat{\mathcal{C}}_{n}\left(q^{-M-1}, q\right)$, where $q$ is a primitive $4 \ell$-th root of unity. It is not hard to show that $a_{\mu^{\prime}}^{\lambda^{\prime}}(-M, \ell)$ is the multiplicity of the simple $\hat{\mathcal{C}}_{n}\left(q^{-M-1}, q\right)$-module labeled by $\lambda$ in the module $U_{n, \mu}$. This second interpretation can be fairly easily proven if one takes for $U_{n, \mu}$ the space of all highest weight vectors of weight $\mu^{\prime}$ in $V^{\otimes n}$ for a suitable integral form of $U_{q} \mathfrak{s} p_{2 m}$, using Lusztig's canonical bases; see [Hu.

5.4. Decomposition matrices for $\hat{\mathcal{C}}_{n}\left(q^{-N-1}, q\right), N$ even. The methods in the last section only work for the image of the representation of $\hat{\mathcal{C}}_{n}\left(q^{-M-1}, q\right)$ in $\operatorname{End}\left(V^{\otimes n}\right)$. Using level-rank duality, Lemma 2.5, we can extend this to the full algebra for all but finitely many $q$.

Theorem 5.5. The decomposition matrix of $\hat{\mathcal{C}}_{n}\left(q^{-N-1}, q\right)$ with $N>0$ even, for diagrams not fixed by any reflection in $W\left(B_{\infty}\right)$ is given by $\left(a_{\mu}^{\lambda}(-N)\right)$, with $a_{\mu}^{\lambda}(-N)$ as in Lemma [5.2, for all but finitely many values of $q$.

Proof. It has been shown that the algebras $\hat{\mathcal{C}}_{n}(r, q)$ are cellular; see XXi]. This means, in particular, that any simple $\hat{\mathcal{C}}_{n}(r, q)$-module is obtained as a quotient of $U_{n, \mu}$ with respect to the annihilator ideal of a certain bilinear form $($,$) . In$ particular, its dimension is equal to the rank of the matrix $\left(\left(b_{i}, b_{j}\right)\right)$ for a certain basis $\left(b_{i}\right)$ of $U_{n, \lambda}$. If $r=q^{-N-1}$, for $N$ positive and even, the entries of this matrix are Laurent polynomials in $q$, and so are all its minors; hence its rank is the same for all but finitely many values of $q$. Using Lemma 2.5, we know that $\hat{\mathcal{C}}_{n}\left(q^{-N-1}, q\right) \cong \hat{\mathcal{C}}_{n}\left(q^{-(2 \ell-N-2)-1}, q\right)$, for $q$ a primitve $4 \ell$-th root of unity. Hence, for $\ell$ sufficiently large, we have $2 \ell-N-2>n$ and we can use Theorem 5.3 to determine the decomposition matrix of these algebras. It follows from this and Lemma 5.2 that $a_{\mu}^{\lambda}(-(2 \ell-N-2), \ell)=a_{\mu}^{\lambda}(-N)$ for large enough $\ell$. Hence, using Lemma 2.5. we have for the multiplicity of a simple $\hat{\mathcal{C}}_{n}\left(q^{-N-1}, q\right)$ module labeled 
by $\lambda^{\prime}$ in $U_{n, \mu^{\prime}}$ that

$$
a_{\mu}^{\lambda}(-N, \ell)=a_{\mu^{\prime}}^{\lambda^{\prime}}(-(2 \ell-N-2), \ell)=a_{\mu^{\prime}}^{\lambda^{\prime}}(-N),
$$

for sufficiently large $\ell$. As this holds for infinitely many values of $q$, it must hold for all but finitely many values of $q$, by the first part of the proof.

Remark 5.6. 1. It is well known how to calculate the coefficients $a_{\mu}^{\lambda}(-N)$ also for Young diagrams $\lambda$ which are fixed by a reflection of $W\left(B_{\infty}\right)$. They can be computed by a slight generalization of the Kazhdan-Lusztig algorithm; see e.g. GW.

2. Our proof above can be easily adapted to the orthogonal case, i.e., for the algebras $\hat{\mathcal{C}}_{n}\left(q^{N-1}, q\right)$ with $N>0$, assuming an analog of the result in $\mathrm{Hu}$ for orthogonal quantum groups. Here the results would be expressed in terms of parabolic Kazhdan-Lusztig polynomials for the reflection groups $S_{\infty} \subset W\left(D_{\infty}\right)$.

3. We expect that we have the same coefficients $a_{\mu}^{\lambda}(-N)$ also for the Brauer algebra $\mathcal{C}_{n}(-N)=\lim _{q \rightarrow 1} \hat{\mathcal{C}}_{n}\left(q^{N-1}, q\right)$. In particular, this should be compatible with the results in $\mathrm{Mt}$.

4. It is easy to find a categorical interpretation of our results, mimicking the theory of tilting modules and fusion categories in the context of quantum groups: It is well known how to define a category $\operatorname{Rep}(O(x))$ depending on a paramter $x$; see e.g. [D. In our context, this could also be done by applying the idempotent construction to Brauer algebras (see e.g. TW2, ThW] for similar constructions in connection with the algebras $\left.\hat{\mathcal{C}}_{n}(r, q)\right)$. Then the category $\mathcal{C}(N)$ derived from the idempotents of the algebras $\mathcal{C}_{n}(N)$ corresponds to the category of tilting modules. It contains the category $\operatorname{Rep}(O(N))$ (for $N>0$ ) and $\operatorname{Rep}(\operatorname{Sp}(|N|))$ (for $N<0$ ) as quotients, similarly, as the fusion categories are quotients of the category of tilting modules of quantum groups.

\section{ACKNOWLEDGEMENT}

I would like to thank the referee for his/her remarks which led to an improved presentation of the material.

\section{REFERENCES}

[A] Andersen, H.H., Tensor products of quantized tilting modules. Comm. Math. Phys. 149 (1992), no. 1, 149-159. MR.1182414 (94b:17015)

[AP] Andersen, H.H., Paradowski, J., Fusion categories arising from semisimple Lie algebras. Comm. Math. Phys. 169 (1995), no. 3, 563-588 MR1328736 (96e:17026)

[AF] Anderson, F.W. and Fuller, K.R., Rings and categories of modules, Springer-Verlag (1974). MR0417223(54:5281)

[Bl] Blanchet, Ch., Hecke algebras, modular categories and 3-manifolds quantum invariants. Topology 39 (2000), no. 1, 193-223. MR.1710999(2000i:57020)

[BB] Beliakova, A. and Blanchet, Ch., Modular categories of types B, C and D. Comment. Math. Helv. 76 (2001) 467-500 MR1854694(2003e:57050)

[Br] Brauer, R., On algebras which are connected with the semisimple continuous groups, Ann. of Math. 63 (1937), 854-872.

[D] Deligne, P., La sèrie exceptionnelle de groupes de Lie. (French) [The exceptional series of Lie groups] C. R. Acad. Sci. Paris Sér. I Math. 322 (1996), no. 4, 321-326. MR.1378507 $(96 \mathrm{~m}: 22012)$

[EW] Enright, Th. and Willenbring, J., Hilbert series, Howe duality and branching for classical groups. Ann. of Math. (2) 159 (2004), no. 1, 337-375. MR.2052357 (2005d:22013)

[CdVM] Cox, A., De Visscher, M., Martin, P., Alcove geometry and a translation principle for the Brauer algebra. arXiv:0807.3892 
[FMS] Di Francesco, Ph., Mathieu, P., Sénéchal, D., Conformal Field Theory, Springer, 1997 MR:1424041 (97g:81062)

[GL] Graham, J.J., Lehrer, G. I., Cellular algebras, Invent. Math. 123 (1996), no. 1, 1-34. MR.1376244 (97h:20016)

[GH] Goodman, F.M., Hauschild Mosley, H., Cyclotomic Birman-Wenzl-Murakami algebras. I. Freeness and realization as tangle algebras. J. Knot Theory Ramifications 18 (2009), no. 8, 1089-1127 MR2554337(2010j:57014)

[GW] Goodman, F. Wenzl, H., A path algorithm for affine Kazhdan-Lusztig polynomials. Math. Z. 237 (2001), no. 2, 235-249. MR1838309 (2002i:20006)

$[\mathrm{Hu}] \mathrm{Hu}, \mathrm{J} ., \mathrm{BMW}$ algebra, quantized coordinate algebra and type $C$ Schur-Weyl duality, Representation Theory 15 (2011), 1-62.

[Hm] Humphreys, J.E., Reflection groups and Coxeter groups. Cambridge Studies in Advanced Mathematics, 29. Cambridge University Press. MR.1066460 (92h:20002)

[KL] Kazhdan, D., Lusztig, G., Tensor structures arising from affine Lie algebras. I, II. J. Amer. Math. Soc. 6 (1993), no. 4, 905-947, 949-1011. MR1186962 (93m:17014)

[Kc] Kac, V., Infinite-dimensional Lie algebras, 3rd edition, Cambridge University Press. MR:1104219 (92k:17038)

[KP] Kac, V., Peterson, D., Infinite-dimensional Lie algebras, theta functions and modular forms. Adv. in Math. 53 (1984), no. 2, 125-264. MR750341 (86a:17007)

[Ks] Kassel, Ch., Quantum groups, Springer. MR.1321145 (96e:17041)

[Ki] King, R., Modification rules and products of irreducible representations for the unitary, orthogonal, and symplectic groups, J. Math. Phys. 12 (1971), 1588-1598. MR0287816 (44:5019)

[KT] Koike, K. and Terada, I., Young-diagrammatic methods for the representation theory of the classical groups of type $B_{n}, C_{n}, D_{n}$. J. Algebra 107 (1987), no. 2, 466-511. MR885807 (88i:22035)

[LR] Leduc, R., Ram, A., A Ribbon Hopf Algebra Approach to the Irreducible Representations of Centralizer Algebras: The Brauer, Birman-Wenzl, and Type A Iwahori-Hecke Algebras, Adv. in Math. 125, 1-94 (1997). MR1427801 (98c:20015)

[Li] Littlewood, D. E., On invariant theory under restricted groups. Philos. Trans. Roy. Soc. London. Ser. A. 239, (1944). 387-417. MR0012299 (7:6e)

[Mac] MacDonald, I., Symmetric functions and Hall polynomials, Oxford University Press MR1354144 (96h:05207)

[Mt] Martin, P., The decomposition matrices of the Brauer algebra over the complex field. arXiv:0908.1500

[MPS] Morrison, S., Peters, E., Snyder, N., Knot polynomial identities and quantum group coincidences, arXiv:1003.0022

[R] Ram, A., A "second orthogonality relation" for characters of Brauer algebras. European J. Combin. 18 (1997), no. 6, 685-706. MR1468338 (98m:20015)

[RW] Ram, A. and Wenzl, H., Matrix units for centralizer algebras J. Algebra. 102 (1992), 378-395. MR.1144939 (93g:16024)

[So1] Soergel, W., Kazhdan-Lusztig polynomials and a combinatoric[s] for tilting modules. Represent. Theory 1 (1997), 83-114 MR.1444322 (98d:17026)

[So2] Soergel, W., Charakterformeln für Kipp-Moduln über Kac-Moody-Algebren, Representation Theory 1 (1997) 115-132 MR.1445716 (98f:17016)

[Su] Sundaram, Sh., Tableaux in the representation theory of the classical Lie groups. Invariant theory and tableaux (Minneapolis, MN, 1988), 191-225, IMA Vol. Math. Appl., 19, Springer, New York, 1990 MR1035496 (91e:22022)

[TbW] Tuba, Imre, Wenzl, Hans, On braided tensor categoriesof type $B C D$. J. Reine Angew. Math. 581 (2005), 31-69. MR2132671 (2006b:18003)

[Tu1] Turaev, V. G., The Yang-Baxter equation and invariants of links. Invent. Math. 92 (1988), no. 3, 527-553. MR939474 (89e:57003)

[Tu] Turaev, V., Quantum invariants, DeGruyter.

[TW1] Turaev, V., Wenzl, H., Quantum invariants of 3-manifolds associated with classical simple Lie algebras. Internat. J. Math. 4 (1993), no. 2, 323-358. MR1217386 (94i:57019)

[TW2] Turaev, V. and Wenzl, H., Semisimple and modular categories from link invariants, Math. Ann. 3-9 (1997) 411-461. MR.1474200 (98j:18012) 
[Wa] Wassermann, A., Operator algebras and conformal field theory. III. Fusion of positive energy representations of $\operatorname{LSU}(N)$ using bounded operators. Invent. Math. 133 (1998), no. 3, 467-538. MR.1645078 (99j:81101)

[W1] Wenzl, H., Hecke algebras of type $A_{n}$ and subfactors, Invent. Math 92, 349-383 (1988). MR936086 (90b:46118)

[W2] Wenzl, H., Quantum Groups and Subfactors of type B, C, and D, Comm. Math. Phys. 133, 383-432 (1990). MR:1090432 (92k:17032)

[W3] Wenzl, H., On the structure of Brauer's centralizer algebras, Ann. of Math., 128, 173-193 (1988). MR951511 (89h:20059)

[W4] Wenzl, H., Braids and invariants of 3-manifolds, Invent. Math. 114, (1993), 235-275. MR:1240638 (94i:57021)

[W5] Wenzl, H., $C^{*}$ tensor categories from quantum groups. J. Amer. Math. Soc. 11 (1998), no. 2, 261-282. MR.1470857 (98k:46123)

[Wy] Weyl, H., The classical groups, Princeton University Press. MR.1488158 (98k:01049)

[Xi] Xi, Ch., On the quasi-heredity of Birman-Wenzl algebras. Adv. Math. 154 (2000), no. 2, 280-298. MR1784677 (2001g:20008)

Department of Mathematics, University of California San Diego, La Jolla CaliforNIA 92093-0112

E-mail address: hwenzl@ucsd.edu 